\title{
Tornadoes in Northern Eurasia: From the Middle Age to the Information Era ${ }^{\mathscr{O}}$
}

\author{
Alexander Chernokulsky, ${ }^{\mathrm{a}}$ Michael Kurgansky ${ }^{\mathrm{a}}$ IgOR MoKhov, ${ }^{\mathrm{a}, \mathrm{b}}$ Andrei Shikhov, ${ }^{\mathrm{c}}$ \\ IGOR AZHIGOV, ${ }^{\mathrm{c}}$ EVGENIYA SELEZNEVA, ${ }^{\mathrm{a}}$ DENIS ZAKHARCHENKO, ${ }^{\mathrm{a}, \mathrm{b}, \mathrm{d}}$ BOGDAN ANTONESCU, ${ }^{\mathrm{e}, \mathrm{f}}$ AND \\ THILO KÜHNE ${ }^{\mathrm{f}}$ \\ ${ }^{a}$ A. M. Obukhov Institute of Atmospheric Physics, Russian Academy of Sciences, Moscow, Russia \\ ${ }^{\mathrm{b}}$ Lomonosov Moscow State University, Moscow, Russia \\ ${ }^{\mathrm{c}}$ Perm State University, Perm, Russia \\ ${ }^{\mathrm{d}}$ The Hydrometeorological Centre of Russia, Moscow, Russia \\ e National Institute of Research and Development for Optoelectronics INOE2000, Ilfov, Romania \\ ${ }^{\mathrm{f}}$ European Severe Storms Laboratory, Wessling, Germany
}

(Manuscript received 29 July 2019, in final form 11 April 2020)

\begin{abstract}
The last comprehensive statistics of tornadoes in northern Eurasia (NE) were published more than 30 years ago. This paper introduces a new database of tornadoes in NE that spans from the tenth century to 2016. The database, compiled using various sources, contains 2879 tornado cases over land and water and includes tornado characteristics. Tornadoes are common for most regions of NE, with a density reaching four cases per $10^{4} \mathrm{~km}^{2}$ in 1900-2016 in some regions. Tornadoes over land have distinct annual and diurnal cycles: they form mostly in May-August, with a maximum in June, and during daytime, with a maximum at 1700-1800 local time. Waterspouts form in all months with a maximum in late summer and mostly at 0900-1300 local time. Most tornadoes are weak and short lived. The Fujita-scale intensity is $\leq \mathrm{F} 1$ for $80 \%$ and $\geq \mathrm{F} 3$ for $3 \%$ out of all rated tornadoes. Half last less than $10 \mathrm{~min}$. The average annual number of all tornadoes over land is around 150 , including 10 and 2 tornadoes with $\geq \mathrm{F} 2$ and $\geq \mathrm{F} 3$ intensity, respectively. Annually, $1-2$ tornadoes lead to casualties and result in 2.9 fatalities and 36.3 injuries. Despite the incompleteness of the dataset, our results show that tornadoes in NE, although being rare, are not as extremely rare as has been thought before. The results illustrate the substantial underestimation of tornado threat by the general public, researchers, and meteorologists, and unambiguously indicate the need for systematic assessments and forecasting of tornadoes by national weather services.
\end{abstract}

\section{Introduction}

Tornadoes are among the most destructive meteorological phenomena that are observed on every continent except Antarctica (Goliger and Milford 1998). Historically, scientists have researched tornadoes widely in North America (e.g., Brooks et al. 1994; Wurman et al. 1996; Verbout et al. 2006; Doswell 2007a; Wurman et al. 2012; Edwards et al. 2013; Schultz et al. 2014; Brooks et al. 2014; Tippett et al. 2015, 2016) where about 1400 tornadoes and 100 tornado-induced casualties are reported each year (NOAA/NWS 2018; NOAA/NWS Storm Prediction Center 2018). Recently, the phenomenon has

Supplemental information related to this paper is available at the Journals Online website: https://doi.org/10.1175/MWR-D-190251.s1.

Corresponding author: Alexander Chernokulsky, a.chernokulsky@ ifaran.ru also received detailed attention in Eurasia (Antonescu et al. 2016). In particular, researchers presented thorough tornado climatologies for individual Asian (Niino et al. 1997; Kahraman and Markowski 2014; Chen et al. 2017) and European countries (e.g., Rauhala et al. 2012; Simeonov et al. 2013; Antonescu and Bell 2015; Taszarek and Brooks 2015; Miglietta and Matsangouras 2018), as well as for the entirety of Europe (Dotzek 2003; Groenemeijer and Kühne 2014). These studies primarily aimed to overcome underestimation of actual tornado number. A conspicuous exception is northern Eurasia. ${ }^{1}$ The latest tornado climatology for this region dates back to 1987 (Snitkovskii 1987), which presented information on 248 tornadoes compiled by the Soviet

\footnotetext{
${ }^{1}$ By northern Eurasia, we mean the territory of 15 countries: Armenia, Azerbaijan, Belarus, Estonia, Georgia, Kazakhstan, Kyrgyzstan, Latvia, Lithuania, Moldova, Russia, Tadzhikistan, Turkmenistan, Ukraine, and Uzbekistan.
} 
National Weather Service. Consequently, the threat of tornadoes in northern Eurasia (NE) may be substantially underestimated.

Tornadoes in NE can occasionally have significant impact. For example, the tornado outbreak on 9 June 1984 resulted in at least 69 fatalities, being among the strongest outbreaks in Europe (Chernokulsky and Shikhov 2018). In the twenty-first century, several severe tornadoes passed across large Russian towns (Kurgansky et al. 2013; Novitskii et al. 2015; Chernokulsky et al. 2017; Romanskii et al. 2018; Shikhov et al. 2019) and attracted the attention of the general public, researchers, and meteorologists. During the last decade, information on a large number of tornadoes with eyewitness accounts has emerged on social networks (Meteodnevnik 2014). Additionally, Shikhov and Chernokulsky (2018) recently discovered dozens of unreported tornadoes in forested regions of Russia. All this points to the incompleteness of current knowledge on tornado occurrence in NE, which can be overcome, in part, by creating an up-to-date tornado climatology using all available sources. Such a climatology is presented in this article.

The article is structured as follows. Section 2 discusses the sources for the new tornado climatology. Section 3 describes the main characteristics of tornadoes in the database. Sections 4-6 present the main results including tornado spatial and temporal variability (section 4), the distribution of tornado characteristics (section 5), and tornado impact (section 6). Section 7 summarizes the results.

\section{Sources for new climatology}

Throughout the paper, we use the word "tornado" to refer to a well-defined phenomenon, namely a rotating column of air that is in contact with both the surface and the mother cloud, with wind strong enough to cause damage to objects. We use the word "case," or "tornado case," to refer to the entire event when one or more tornadoes formed but shared characteristics in the database (see section $3 \mathrm{~d}$ for details). Tornadoes can be reported directly by an eyewitness, or after the event, given their impact (Edwards et al. 2013). In this study, we utilized information from both primary and secondary sources that are described below.

\section{a. Historical tornadoes: Ancient chronicles and regional overviews}

The earliest mentions of tornadoes over NE come from ancient Russian chronicles, so-called codices, like the Novgorodian codex or Laurentian codices. We utilized the Complete Collection of Russian Chronicles (CCRC) (CCRC 1841) that provides the most complete information on Russian historical events, including remarkable weather events, from the tenth to the eighteenth centuries. The word smerch, which is a name for the class of events that include tornadoes, landspouts, and waterspouts, appeared in the Russian language only in the nineteenth century. Therefore, we searched the CCRC for the words vikhor (i.e., whirlwind), and burya (i.e., windstorm), and carefully examined the accompanying description of the damage. However, in many cases, it was impossible to discriminate between tornadoes and other wind-related events (e.g., downburst- or gustfront-induced winds).

Some historical events originate from local overviews that are commonly published in regional magazines (e.g., Chizhikov 1956; Ovchinnikova 2013). These overviews are based on carefully gathered information about past tornado events from fragmented records and tales. They usually provide a description of the associated tornado damage and rarely include other tornado characteristics (e.g., pathlength or width).

We obtained 134 cases (i.e., $4.5 \%$ of all cases), from ancient chronicles and regional overviews (Fig. 1), these being the only sources of data on tornado occurrence before the eighteenth century.

\section{b. News reports}

For more recent events, we utilized scanned newspapers from the Eastview company database (https:// dlib.eastview.com). It is of note that the database contains different numbers of newspapers for various historical periods: only two for the Soviet Era (1918-91), namely Pravda and Izvestiya, and dozens for the modern times. In addition, we used web versions of news agencies to search for tornado reports. Russian-language media were mainly utilized and other-language media (e.g., Ukrainian, Estonian, or Georgian) were used to a lesser extent.

The reports sometimes include eyewitness testimonies, which can directly indicate tornado occurrence, or contain a detailed damage description that helps to estimate the tornado intensity and its path characteristics. More recent news reports occasionally include some visual evidence of tornado-induced damage or the tornado itself. Additionally, news reports provide information on event-related economic loss and casualties.

We treated reports that lacked detailed information with caution. Since tornadoes are rare in Northern Eurasia and not well understood, reporters sometimes apply the word "tornado" (smerch) to other severewind events, like gustfronts or downbursts. In particular, this type of reports appears in the media one or two months after the occurrence of a significant wind event, especially in a relatively big city. In contrast, true tornado events are sometimes described in the media as 


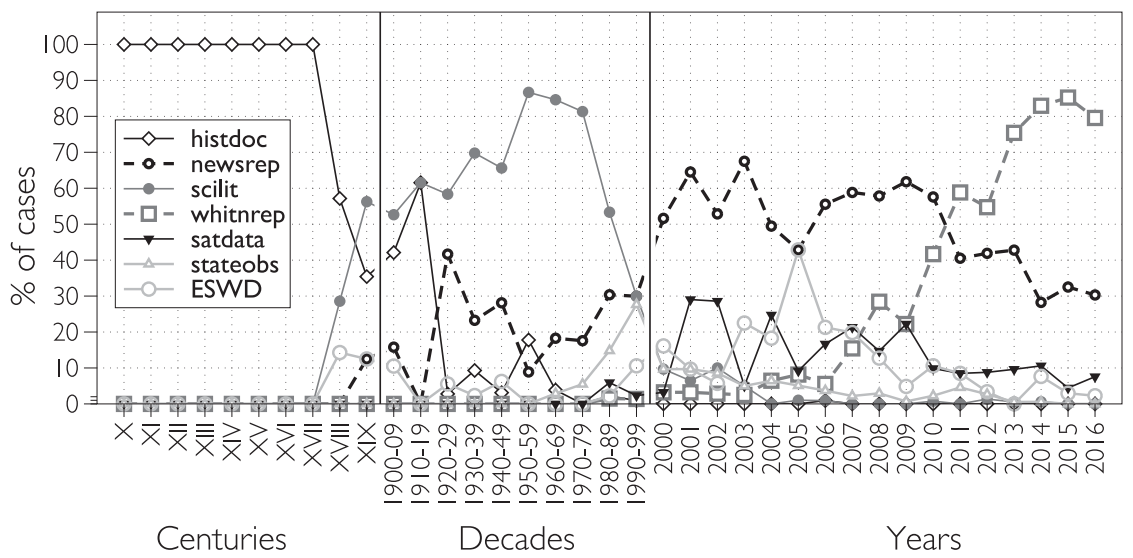

FIG. 1. Percentage of different sources of information on tornado cases for different periods - centuries for the period before the nineteenth century, decades for the twentieth century, and years for the twenty-first century. Note that some cases come from multiple sources; therefore, the sum may exceed $100 \%$. Acronyms on the figure stand for: histdochistorical documents including ancient chronicles and regional overviews, newsrep-news reports, scilit-scientific literature including case studies and existing climatologies, whitnrep-witness photos, videos and reports in the Internet and social networks, satdatasatellite data on forest disturbances, stateobs-meteorological observations and data from national meteorological services, ESWD—-the European Severe Weather Database.

hurricane/hurricane-force wind (uragan). A similar issue was found for Poland (Taszarek and Gromadzki 2017), which is a neighbor to NE.

News reports are one of the main sources of information on tornadoes after 1873 , when a waterspout was mentioned for the first time in a Russian newspaper (i.e., in Niva) (Niva 1874). In total, we used news reports for information on 1138 cases (Fig. 1).

\section{c. Case studies and existing climatologies}

The Moscow F4 tornado of 29 June 1904 (Mikhelson 1904) caught the attention of Russian scientists for tornadoes over land. Early case studies were mostly descriptive and provided information on the associated damage, an estimated wind speed and other characteristics, such as tornado path width and length, rotation direction (e.g., Letzmann 1923; Zykov 1947; Popov 1954; Kolobkov 1957; Peryshkin 1957). They focused on both tornadoes over land and water, particularly on waterspouts over the Black Sea (Starov 1935; Popov 1955). Later, researchers started using synoptic and aerological data to analyze the associated weather conditions and convective instability indices (e.g., Gaigerov 1939; Tomashevich 1952; Prokh and Skotarenko 1971; Vasiliev et al. 1985; Tunegolovetc and Mikhailenko 1998; Chernokulsky et al. 2015; Novitskii et al. 2015). Since the middle of the twentieth century, several researchers in the USSR have compiled information on tornadoes from different sources (e.g., Voznyachuk 1954a,b; Nalivkin 1970; Snitkovskii 1987; Scientific and
Engineering Centre for Nuclear and Radiation Safety 2001; Tooming and Merilain 2004; Marcinoniene 2003; Vashchenko et al. 2018). Based on statistics of 248 cases, Snitkovskii (1987), for the first time, determined four specific synoptic patterns favorable for tornado formation over NE. The list of tornadoes in Scientific and Engineering Centre for Nuclear and Radiation Safety (2001) was used to estimate the wind load on Russian nuclear plants. Recent studies of individual tornadoes used mesoscale modeling (e.g., Novitskii et al. 2016; Romanskii et al. 2018) and attempted to attribute tornadic events to global climate change (e.g., Kurgansky et al. 2013; Chernokulsky et al. 2017).

Tornadoes in different parts of NE are studied with varying degrees of attention. They have received the most attention in Estonia (Antonescu et al. 2016) owing to Letzmann's and Tooming's studies (Letzmann 1923; Tooming and Merilain 2004). Ukranian tornadoes were analyzed thoroughly by Prokh (1983). At the same time, Siberian and Russian Far East tornadoes have received much less attention.

In total, we obtained information on 478 tornado cases from existing climatologies and single case studies (Fig. 1).

\section{d. Witness photos, videos, and reports in the internet and social networks}

Recently, the spread of smartphones with camera capability and the fast mobile internet (ITU 2018) resulted in an explosive growth of photo and video 
material on severe weather events, including tornadoes, in many countries (e.g., Kahraman and Markowski 2014; Antonescu et al. 2016). People have become more aware of such events (Antonescu et al. 2017) and have attempted to relate photos and videos of tornadoes with information on time, place of occurrence, and on associated damage.

Furthermore, Internet fora and social media networks have made it possible to connect a researcher directly with witnesses of events. Beginning in 2011, weather amateurs in Russia and Ukraine have compiled information on tornado events from social media networks (e.g., Tornado list 2013, 2014, 2015, 2016; Meteodnevnik 2014). Since 2013, one of the authors of this article (Azhigov) has interviewed tornado witnesses via social media networks, primarily via the Vkontakte network, to reveal information, such as the exact path and time of tornado occurrence, and damage description. This verification is important since some nontornadic events (e.g., low-level scud clouds) can be mistaken for a tornado because of the limited experience of witnesses (Doswell 2003). Some cases can be fake, such as when people post photos of another event, from another country sometimes, or even draw a funnel in a graphical editor.

In this paper, 1003 cases come directly from witnesses of tornado events, mostly for the period after 2007 (Fig. 1).

\section{e. Satellite data}

The number of tornado reports from all the sources mentioned above depends on the population density (Anderson et al. 2007), which is very low in many NE regions (GPWv4.112018), particularly, in forested areas (Fig. 2a). Dyer (1988) proposed using aerial photographs to look for tornadoes in forested areas that had no eyewitnesses. Shikhov (2013) suggested using satellite data on forest disturbances for the same purpose, while Shikhov and Chernokulsky (2018) developed an approach to obtain characteristics of previously unreported tornadoes in forests using remote sensing data. These authors used Landsat-based Global Forest Change data (Hansen et al. 2013) to look for narrow elongated forest disturbances, the so-called candidates, and verified the tornado-caused nature of these disturbances by revealing cyclonic (counterclockwise) patterns of fallen trees using high-resolution satellite images (see Fig. 2 for several examples). They also used additional information (i.e., Landsat and MODIS satellite images, surface observations, reanalysis, and news reports) to estimate the date of the tornado occurrence. In particular, the sequence of Landsat satellite images narrows the range of potential dates. Reanalysis data help find meteorological conditions that are favorable for tornado formation during those dates, and hence to determine the exact date of the tornado occurrence. Given the known Weibull distribution for tornado pathlengths and widths (Brooks 2004), Shikhov and Chernokulsky (2018) obtained probability estimates of the minimum tornado intensity (see also section 3e). The method allows robust identification of tornadoes with a path width exceeding $60 \mathrm{~m}$ in regions with $>50 \%$ forest coverage. As a result, 105 previously unreported tornadoes in northeast Europe for the period 2000-14 were found (Shikhov and Chernokulsky 2018). We included these cases into our database and applied the same approach to other NE forested regions for the period 2000-16.

We also used satellite data to specify exact coordinates, pathlengths and widths of tornadoes that initially were obtained from other sources and passed through forested areas on their way (Shikhov and Chernokulsky 2018). In particular, we used Landsat images to identify tornado-induced forest disturbances and determine characteristics of tornadoes during the 1984 Ivanovo tornado outbreak (Chernokulsky and Shikhov 2018).

In total, we used satellite data to determine the characteristics of 275 tornadoes mostly after 2000, either specifying known events or revealing previously unreported ones.

\section{f. Meteorological observations and other data}

Tornadoes only rarely pass close to meteorological stations at the moment they report current weather conditions. These cases are included in observational databases and can be separated from other whirlwind events (i.e., dust devils and snowstorms) given additional information (e.g., precipitation, temperature, and cloud types). We used information from more than 500 stations for the 1966-2016 period that were collected in the Russian Institute for Hydrometeorological Information (Bulygina et al. 2014). In addition to direct observations, we used information from lists of severe weather events compiled by national hydrometeorological services (HMS). Particularly, we used information from the Ukrainian and Russian HMS. A total of 111 cases were obtained from these sources (Fig. 1).

Whenever possible, we cross-checked all aforementioned sources against each other. Often, we used multiple sources for the same tornado event. We involved auxiliary data such as ERA-Interim reanalysis (Dee et al. 2011), Meteosat satellite data, or Russian weather radar network (Dyaduchenko et al. 2014) to specify the date of an event if this information was missing from the initial report.

\section{g. European Severe Weather Database}

The European Severe Weather database (ESWD) presents information on NE tornadoes (Groenemeijer and Kühne 2014). The ESWD is maintained by the 

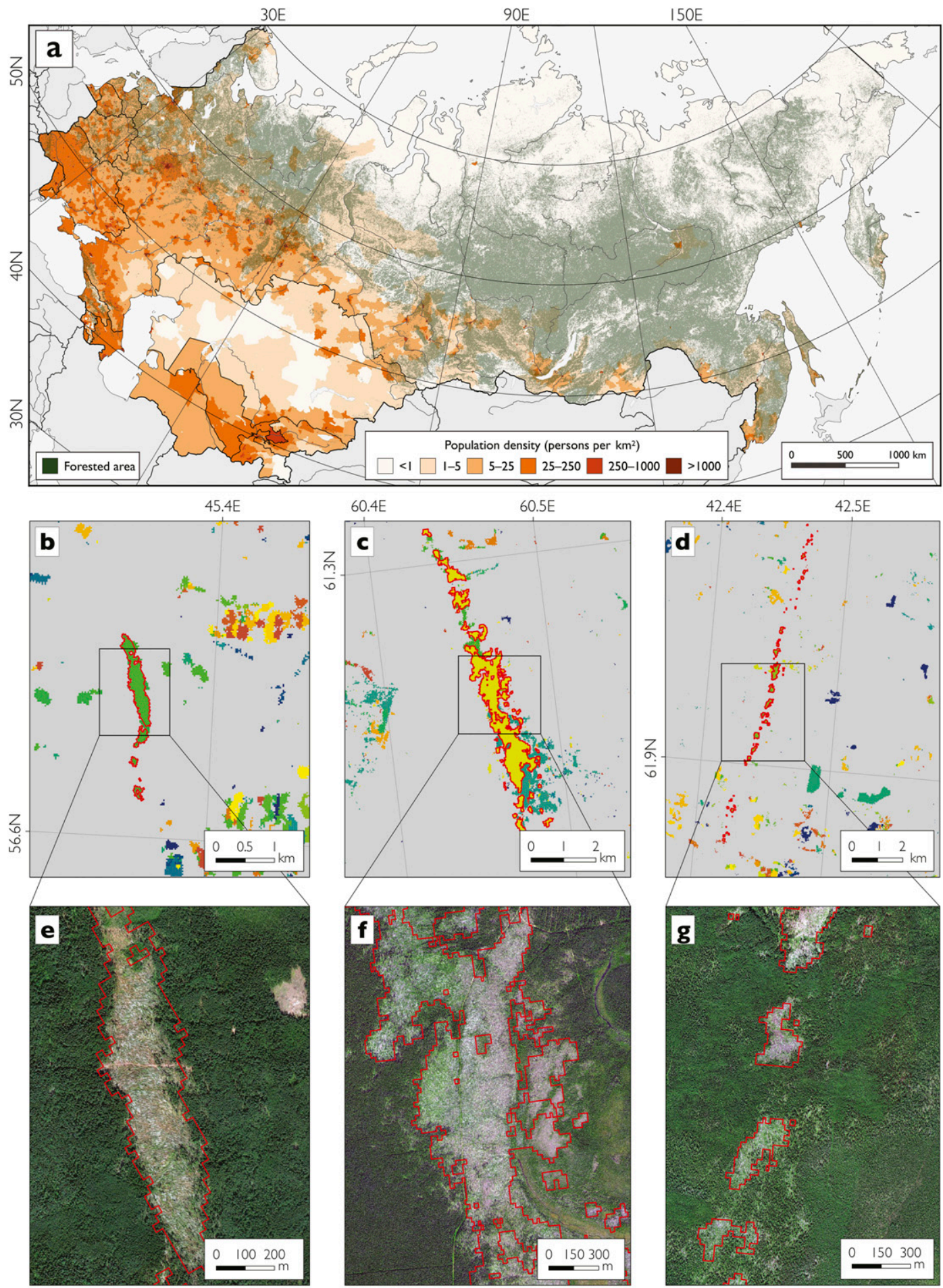

Forest loss year

2016

$\Xi$ tornado damaged area

FIG. 2. (a) Forested area in northern Eurasia (Arino et al. 2012) combined with population density (GPWv4.11 2018); (b)-(g) Examples of the identification of tornado damage tracks in forested areas using (b)-(d) the Global Forest Change data and (e)-(g) high-resolution images from publicly-available web services for the tornado tracks in (b),(e) Nizhniy Novgorod region, 2011, (c),(f) Sverdlovsk region, 2007, and (d),(g) Arkhangelsk region, 2009. Forest loss year is given according to the Global Forest Change data (Hansen et al. 2013). 
European Severe Storms Laboratory (ESSL), which was founded in 2006 with the aim of bringing together researchers and forecasters from different European countries with an interest in severe weather (Dotzek et al. 2009). Since 2018, the ESWD also covers central Asian countries. The ESWD contains more than 100000 individual severe weather reports (Groenemeijer et al. 2017). Data come to the ESWD from voluntary observers, ESSL staff, and several national weather services (none from NE) (Groenemeijer et al. 2017). In NE, this activity is purely voluntary and nonsystematic, which leads to general underreporting of severe weather events (Groenemeijer and Kühne 2014; Groenemeijer et al. 2017).

For the period before 2016, the ESWD contains information on 1851 tornado cases in NE. Recent information came mostly through the public ESWD web interface and was added by a few volunteers who mostly provided direct witness reports (section $2 \mathrm{~d}$ ). The ESSL staff added to the ESWD the majority of cases before 2014 through utilizing case studies, existing climatologies, news reports, and lists from HMS.

Translation issues can be additional source of errors. Of the 1851 cases in the ESWD, we included only 1474 in our database with 1180 cases being revised and 294 utilized as they were. Of the rejected cases, 131 have been reclassified into "severe wind gusts" or "hail" events, while 246 cases have been removed from the ESWD (e.g., literary metaphors, forecast warnings, etc). All changes have been subsequently implemented into the ESWD database.

\section{Database description}

\section{a. Degree of event certainty}

We defined the certainty of a tornado event based on the credibility of the sources. We assigned one of four degrees of certainty (i.e., high, medium, low, and very low) to each event (Table 1). These degrees can be expressed as extremely likely ( $>95 \%$ likelihood of occurrence), likely $(66 \%-95 \%)$, about as likely as not $(33 \%-66 \%)$, and unlikely $(<33 \%)$; however, this division into degrees is more subjective than robustly quantitative.

We assigned a high degree of certainty to 1409 events. These events have highly credible sources like scientific papers, satellite data, eyewitness reports that accompanied by photos/videos of the vortex itself (Table 1). A medium degree of certainty was assigned to 835 events. These events were reported by witnesses but lacked photo or video evidence and did not have documented tornado-related impact, or in turn, had impact but were not accompanied with a witness report. Events with low
(419) and very low (216) certainty mostly came from news reports or ancient chronicles, that were not accompanied by necessary details (Table 1 ).

We obtained most of our results using events with the high and medium degrees of certainty; however, we included both lower categories into the database. Further improvement of the database, for instance with the use of weather radar data, may help to confirm or, instead, refute the tornadic nature of the latter events. However, for now, the routine usage of the Russian meteorological radar data is difficult due to access restrictions.

\section{b. Date and time of the event}

Information on the relevant Gregorian calendar dates accompanies all events. For historical events, we recalculate dates properly from the Byzantine calendar for tornadoes before the eighteenth century and from the Julian calendar for events from 1700 to 1918.

Together with a date, each case in the database has information on UTC and local time. If a source provided event time, it was usually local time. We recalculated it to UTC timing according to governmental laws on time zones for a particular year and a particular country. For instance, in the Russian Empire (i.e., before July 1919) the apparent solar time was used, while in June 1930 new time zones were implemented (adding an extra hour, so-called decree time). For historical overviews, we assumed that the time is relevant for an event year.

We indicated time accuracy for each tornado case. It varies from $1 \mathrm{~min}$ (i.e., the time of an event is known precisely) to 50 years. Timing accuracy increases in time from 1 year on average in the Middle Ages to a dozen minutes in the twenty-first century except for satellitederived tornadoes in forested areas that have an accuracy from a few days to a few weeks (Shikhov and Chernokulsky 2018). In summary, our database includes 1169 cases with time accuracy $\leq 30 \mathrm{~min}, 1835$ cases with accuracy $\leq 3 \mathrm{~h}$, and 2416 cases with accuracy $\leq 1$ day (Table 2).

\section{c. Location of the event and underlying surface}

An event in the database has geographic coordinates (i.e., latitude and longitude) with specified spatial accuracy. The accuracy varies from $10 \mathrm{~m}$ to $200 \mathrm{~km}$ (i.e., from a precise location to that which is known very roughly). The latter is especially characteristic for historical tornadoes. Spatial accuracy is $\leq 1 \mathrm{~km}$ for 1840 cases, while only 209 cases have accuracy $>10 \mathrm{~km}$.

We also provided information on the country of event together with an administrative unit, as of 2019, and on the closest settlement or damaged settlement(s) whenever possible. For tornadoes over water, we indicated the name of the body of water. 


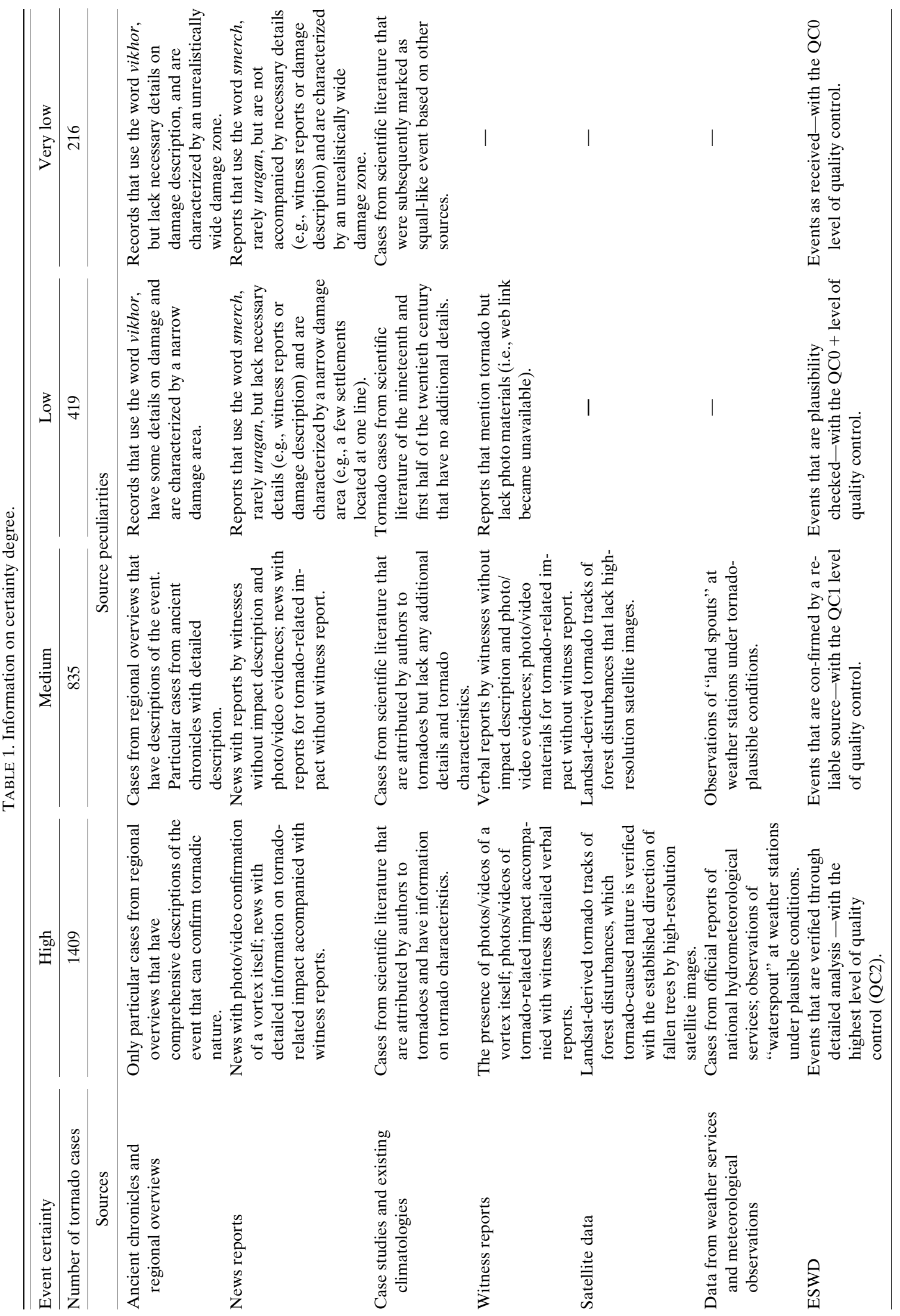


TABLE 2. Summary information on the database for northern Eurasia tornadoes. "All" are events with all degrees of certainty; $H+M$ are events with the high and medium degrees of certainty only.

\begin{tabular}{|c|c|c|c|}
\hline \multicolumn{2}{|c|}{ Characteristics } & \multicolumn{2}{|c|}{ No. of events } \\
\hline \multirow[t]{2}{*}{ Total No. of } & Cases & \multicolumn{2}{|c|}{2879} \\
\hline & Tornadoes & \multicolumn{2}{|c|}{3186} \\
\hline \multirow[t]{6}{*}{ Case certainty degree } & High & \multicolumn{2}{|c|}{1409} \\
\hline & Medium & \multicolumn{2}{|c|}{835} \\
\hline & Low & \multicolumn{2}{|c|}{419} \\
\hline & Very low & \multicolumn{2}{|c|}{216} \\
\hline & & \multicolumn{2}{|c|}{ Event certainty degree } \\
\hline & & All & $H+M$ \\
\hline \multirow{6}{*}{$\begin{array}{l}\text { Timing accuracy } \\
\text { (for cases) }\end{array}$} & $\leq 5 \mathrm{~min}$ & 408 & 384 \\
\hline & $(5-60] \mathrm{min}$ & 1067 & 880 \\
\hline & $(1-3] \mathrm{h}$ & 360 & 239 \\
\hline & $(3-24] \mathrm{h}$ & 581 & 399 \\
\hline & (1-7] days & 92 & 61 \\
\hline & $>1$ week & 371 & 281 \\
\hline \multirow{3}{*}{$\begin{array}{l}\text { Spatial accuracy } \\
\text { (for cases) }\end{array}$} & $\leq 1 \mathrm{~km}$ & 1840 & 1490 \\
\hline & $(1-10] \mathrm{km}$ & 904 & 639 \\
\hline & $>10 \mathrm{~km}$ & 135 & 115 \\
\hline \multirow{5}{*}{$\begin{array}{l}\text { Underlying surface } \\
\text { (for cases) }\end{array}$} & Land only & 2121 & 1567 \\
\hline & Water only & 571 & 521 \\
\hline & Water to land & 119 & 100 \\
\hline & Land to water & 8 & 6 \\
\hline & Undefined & 60 & 50 \\
\hline \multirow[t]{3}{*}{ No. of tornadoes per case } & 1 & 2707 & 2089 \\
\hline & 2 & 116 & 100 \\
\hline & $\geq 3$ & 56 & 55 \\
\hline \multirow{6}{*}{$\begin{array}{l}\text { Fujita scale intensity } \\
\text { for tornado cases (except } \\
\text { for "water only") }\end{array}$} & F0 & 498 & 414 \\
\hline & $\mathrm{F} 1$ & 897 & 735 \\
\hline & $\mathrm{F} 2$ & 312 & 272 \\
\hline & F3 & 58 & 52 \\
\hline & $\mathrm{F} 4$ & 4 & 4 \\
\hline & Unrated & 539 & 246 \\
\hline \multirow[t]{6}{*}{ Tornado cases with defined characteristics } & Pathlength & 574 & 542 \\
\hline & Path mean width & 479 & 465 \\
\hline & Path maximum width & 518 & 503 \\
\hline & Movement direction & 545 & 507 \\
\hline & Rotation & 206 & 203 \\
\hline & Duration & 318 & 293 \\
\hline \multirow[t]{2}{*}{ Cases with } & Casualties & 114 & 79 \\
\hline & Injuries & 146 & 121 \\
\hline
\end{tabular}

We categorized tornadoes by underlying surface and found that 2121 cases had their entire lifetime over land and 571 cases over water. We used the terms "tornado over water" and "waterspout" as synonyms (Rauhala et al. 2012). In addition, we discovered 127 cases of tornadoes crossing both land and water, including 119 passing from water to land and eight from land to water. We failed to determine confidently the underlying surface for 60 cases.

\section{d. Number of tornadoes}

Sometimes, several tornadoes are observed simultaneously, which is more common for tornadoes over water than over land. For instance, witnesses reported 15 simultaneous waterspouts on 22 July 1928 in the Baltic Sea (Tooming and Merilain 2004). Sometimes, the lack of information prevents assigning unique timing and location for each tornado that was simultaneously observed within one event. We combined such tornadoes into one case and provided information on the number of tornadoes that occurred (Table 2). This is in line with the approach used previously (e.g., Rauhala et al. 2012; Kahraman and Markowski 2014; Taszarek and Brooks 2015). We aimed to separate cases with multiple tornadoes into isolated cases wherever possible, as when sources provide the specific time of formation of 
different tornadoes, or satellite data provide exact coordinates of each tornado track.

\section{e. Tornado intensity}

Tornado intensity is an essential characteristic for proper tornado risk assessment (Edwards et al. 2013). We used the Fujita scale (F scale) (Fujita 1981) for tornado intensity rating. In addition to the conventional six categories, from F0 to F5, we assigned the "unrated" category to some events, as was suggested in Doswell et al. (2009) and implemented in many European tornado climatologies (e.g., Rauhala et al. 2012; Groenemeijer and Kühne 2014; Antonescu and Bell 2015; Taszarek and Brooks 2015). The number of unrated tornadoes is especially large for events with low- and very-low certainty (i.e., 67\%), and relatively small for events with medium and high certainty (i.e., around $10 \%$ ) (Table 2). For consistency with other European studies, we used the F scale instead of the enhanced Fujita (EF) scale, which was implemented in the United States (TTU 2004; Doswell et al. 2009; Edwards et al. 2013), or a construction-independent wind speed such as the E scale presented by Dotzek (2009). Proper usage of EF scale outside of the United States requires proposing and validating damage indicators and degrees of damage that account for local construction practices (Doswell et al. 2009). It has been done for Japan (Okada et al. 2015) and has been suggested for other regions (ESSL 2018), but not yet performed for NE.

We estimated tornado intensity based on texts, videos, and photographs of damage, including eyewitness accounts and rare ground damage surveys. The damage descriptions provided by Bunting and Smith (1993) and Doswell (2003) guided the estimate of intensity. For every case, we concentrated on any descriptions of damage to engineered structures, such as water tanks, and of other extraordinary features (e.g., overturned railcar, removal of pavement etc.), which help to limit F-scale estimates to specific rating(s) (Doswell 2003; Doswell et al. 2009). We used information from Feuerstein et al. (2011) for clarification on assignment based on tornado-related damage to vegetation and construction characteristic to Europe. Lack of precise information on details led to the assigning of a range of possible F-scale values (e.g., F1-2), and not a single F-scale value for 222 cases. A similar approach was applied previously by Taszarek and Gromadzki (2017). Later in the paper, these events are used with the lower rating assigned.

Another approach was used to estimate the intensity of tornadoes obtained using only satellite data (Shikhov and Chernokulsky 2018). We gave these estimates in probabilistic terms based on information on tornado pathlength and maximum width. Brooks (2004) derived Weibull distribution parameters that link tornado intensity with pathlength and maximum width for the United States. Although the exact rating is unachievable (Brooks 2004), it is possible to determine a minimal intensity rating given a particular probability level (Shikhov and Chernokulsky 2018). A tornado with given length and width, which has been determined with sufficiently accuracy from satellite data, was assigned an intensity no less than a particular rating (e.g., no less than $\mathrm{F} 2$ with $90 \%$ probability). It was recorded as " $\geq \mathrm{F} 2$ " in the database. But for the analysis, we applied this minimal rating as the estimated $\mathrm{F}$ rating (i.e., $\mathrm{F} 2$ instead of $\geq \mathrm{F} 2$ ). This is somewhat in contrast to the original definition where the assigned $\mathrm{F}$ rating corresponds to the maximum damage along the path (Fujita 1981).

One should keep in mind several issues with the $\mathrm{F}$ scale. The $\mathrm{F}$ scale is a damage scale rather than an intensity scale (Doswell and Burgess 1988; Doswell et al. 2009). The dependence of damage on things other than the wind speed (e.g., construction practice, building integrity and resistance, flying debris, wind fluctuation, and windload duration) may lead to uncertainties in rating estimates (Doswell and Burgess 1988; Doswell 2003; Doswell et al. 2009; Edwards et al. 2013). Doswell and Burgess (1988) speculated that the F rating of many events is inappropriate even in the United States (by one or two categories). Moreover, the F scale is based on a primary indicator-a typical for U.S. wood frame home. It is not clear how exactly it is associated with buildings in NE, especially in rural areas. It is even more problematic to estimate damage for historical events. Subjectivity of an expert who estimates $\mathrm{F}$ rating may influence the result as well (Doswell and Burgess 1988; Edwards et al. 2013). In this paper, the first author made the intensity estimates for the vast majority of cases. For some difficult marginal cases, the intensity was estimated collectively. Therefore, the database lacks spatial and temporal inhomogeneities that might appear when several researchers estimate intensity of tornadoes for different parts and periods within the database (Doswell and Burgess 1988).

\section{f. Other tornado characteristics}

Wherever possible, we included information on tornadoes with details on tornado pathlength, maximum and mean width, tornado movement and rotation direction, tornado duration. Around $15 \%-20 \%$ cases have information on tornado movement direction and path characteristics, and around $10 \%$ on rotation and duration (Table 2). The percentage is larger for more certain events. 
The lack of routine tornado damage surveys in NE regions results in approximate estimates of tornado path width and length. For recent cases, we evaluated tornado pathlength and width based on eyewitness and damage reports or satellite data in damaged forests (Shikhov and Chernokulsky 2018). The pathlength was usually underestimated, since it was difficult to identify the exact place of tornado formation and decay. For historical tornadoes, we took most estimates of path width and length directly from previous climatologies (e.g., from Snitkovskii 1987). However, these estimates are likely biased. Chernokulsky and Shikhov (2018) showed for the Ivanovo outbreak that past databases tend to underestimate maximum path width and overestimate paths length (for very long tornado tracks). Doswell and Burgess (1988) showed that tornadoes with very long paths are often a sequence of short-track tornadoes. They proposed $8-16 \mathrm{~km}$ as a threshold for length of gaps in the damage paths of continuous but "skipping" tornadoes (Doswell and Burgess 1988). We used an $8-\mathrm{km}$ threshold to separate a skipping tornado from consecutive tornadoes. Nevertheless, we did it only when we had enough information, for instance for satellite-derived tornadoes in forest.

We determined the tornado movement direction from information in witness or/and damage reports. Since tornadoes do not usually move along a straight line, the direction was defined between the first and the last damaged locations. Rotation was determined primarily using witness reports, or occasional damage surveys (e.g., Kolobkov 1957). The direction of rotation was also determined by the direction of the fall of trees, obtained from high-resolution satellite images. The duration was estimated based on witness reports, and, for some cases, by comparing the time the tornado passed different settlements. For historical events, duration estimates were taken directly from existing climatologies. As for tornado pathlength, these can be overestimates due to considering a sequence of short-lived tornadoes as a single long-lived tornado. They should be treated with caution.

\section{g. Additional information on tornado impact and description}

If available, we provided additional information including the number of injures, fatalities, a brief description of tornado-related damage, a short narration of eyewitness reports, and economic loss from the event. Economic losses are reported as at the time of the event in the currency in use at that time and location. Additionally, each case contains information on a source.

The database with all 2879 cases accompanies the paper as a separate CSV file (see the online supplemental material).

\section{Spatial and temporal distribution}

\section{a. Year-to-year changes}

Tornado reports in NE vary substantially in time and show a marked increase over recent decades. The earliest tornado in NE dates to 979, although it, not surprisingly, is of low certainty (Fig. 3a). The first tornado with medium certainty occurred in 1143 (Fig. 3b). Subsequently, several tornado cases are mentioned in every century, usually with one event per year. In the Middle Ages, the fifteenth century had the largest number of tornadoes (27 cases) with four years having two events in a year (see also Lyakhov 1987). Since the eighteenth century, the annual number of reported tornado cases shows a steady increase from 1-2 in the eighteenth century to 10-20 by the end of the twentieth century. An increasing amount of information has aided the continued increase in reported tornadoes in recent years. The total annual number of reported tornadoes over land is approximately one hundred during the last decade. Many European countries experienced a recent increase of tornado reports (e.g., Rauhala et al. 2012; Taszarek and Brooks 2015). The ESWD shows the maximum number of European tornadoes in 2006 (Groenemeijer and Kühne 2014), which is not the case in NE. It is of note, however, that the maximum of the reclassified ESWD cases occurs in 2005-06 (see section 2g). Instead, the maximum annual number of tornadoes over land in NE happened in the final year of the analyzed period, 2016, with 172 cases. Weak tornadoes display the largest increase over the recent decades, which very likely points to the incompleteness of the database.

The number of reports of significant and intense tornadoes, (intensity $\geq F 2$ and $\geq F$ 3, respectively), increases as well (Fig. 3). The first significant and intense tornadoes with low certainty occurred in the years 1149 and 1280 , respectively. At the same time, the first significant and intense tornadoes with medium certainty date back to 1300 and 1301. Afterward, at least 1-3 significant tornadoes occurred every century. With the increase of sources (Fig. 1), the number of significant and intense tornadoes grow to 12 and 6 in the nineteenth century and to 155 and 28 in the twentieth century. More than two intense tornadoes happened within tornado outbreaks in the central regions of Ukraine (Prokh and Skotarenko 1971) in 1969 and in the central part of European Russia in 1984 (Vasiliev et al. 1985; Finch and Bikos 2012; Chernokulsky and Shikhov 2018). In the 21st century, the annual rate of significant tornadoes is $10 \pm 4$. Almost every year, one or two intense tornadoes occur as well (including three F3 tornadoes in 2012). The lack of increase in the number of significant and intense tornadoes is 

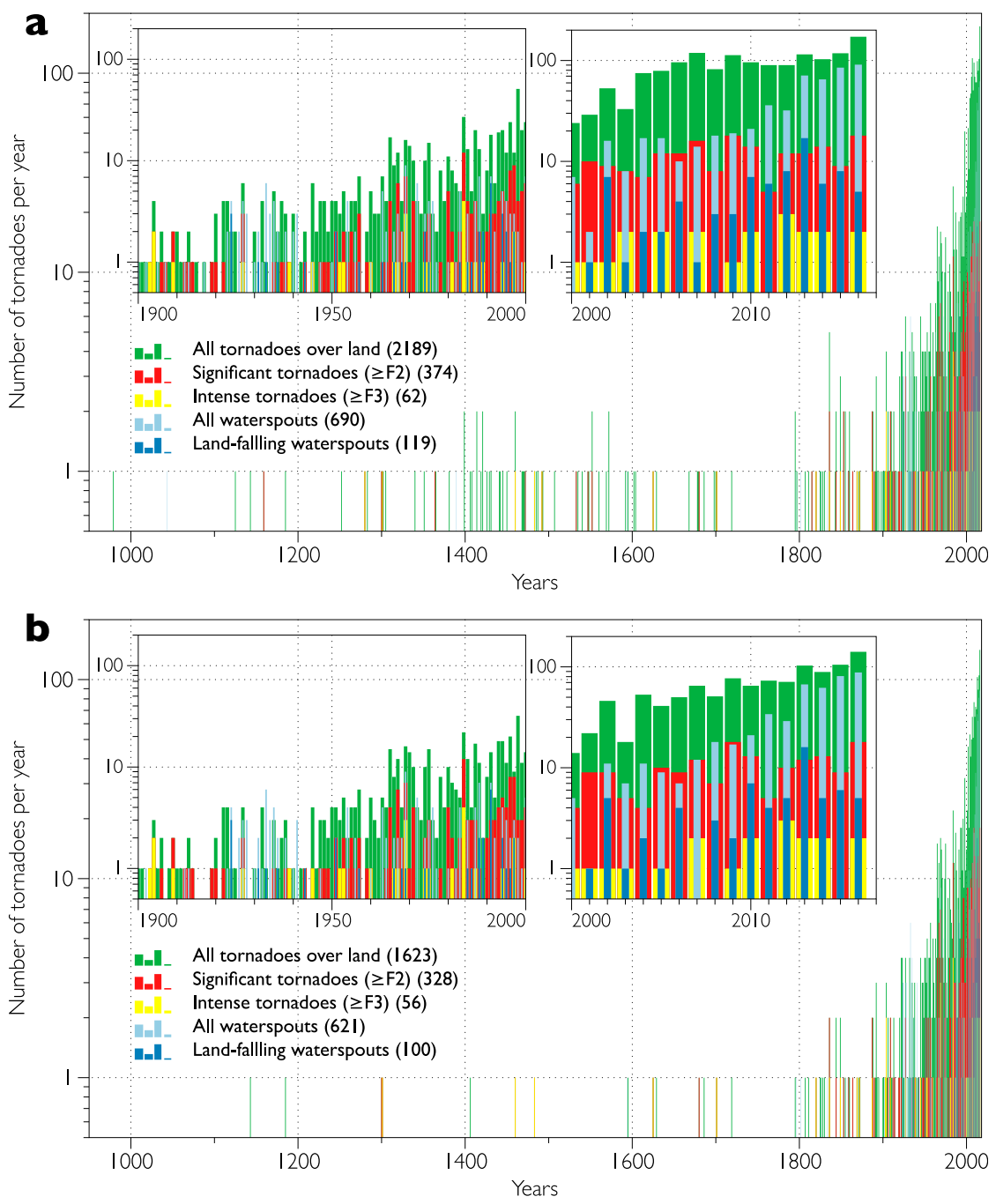

FIG. 3. Number of tornado cases per year for the entire period for (a) cases with any event certainty and (b) cases with only high and medium event certainty. Two insets in each subplot display more detailed changes for the twentieth and twenty-first centuries. Numbers in brackets show the total number of cases for the entire period. Land-to-water cases and cases with undefined underlying surface are treated as tornadoes over land. Note that the logarithmic scale is used.

in contrast to the increase of all tornadoes. Presumably, it means that the recent numbers of significant and intense tornadoes are the reasonable upper bounds on the numbers that actually detectable given the variety of sources used. This is generally in line with the findings of Brooks and Doswell (2001) and Verbout et al. (2006) who showed more temporal consistency of data for intense tornadoes, especially for those that had a big impact.

The number of waterspouts is likely underreported. It increased in the twenty-first century up to $70-90$ per annum recently. The abrupt rise happened in 2013 when information from social media networks started to be thoroughly utilized. Around $10 \%$ of waterspouts made landfall. In the most recent decade, 8-15 landfalling waterspouts were reported each year. It is of note that temporal inhomogeneity of the number of data sources results only in positive trends of tornado occurrence, over land or water, while the small sample size of these relatively rare events determines year-to-year variations (Doswell 2007b).

\section{b. Spatial distribution}

Tornadoes are common for most regions of NE, especially for its European part (Fig. 4, Table 3). The density of reports of all tornadoes and that of significant tornadoes reaches 4 and 2 cases per $10^{4} \mathrm{~km}^{2}$, respectively (Fig. 5). This is 100 and 10 times less than in the United 


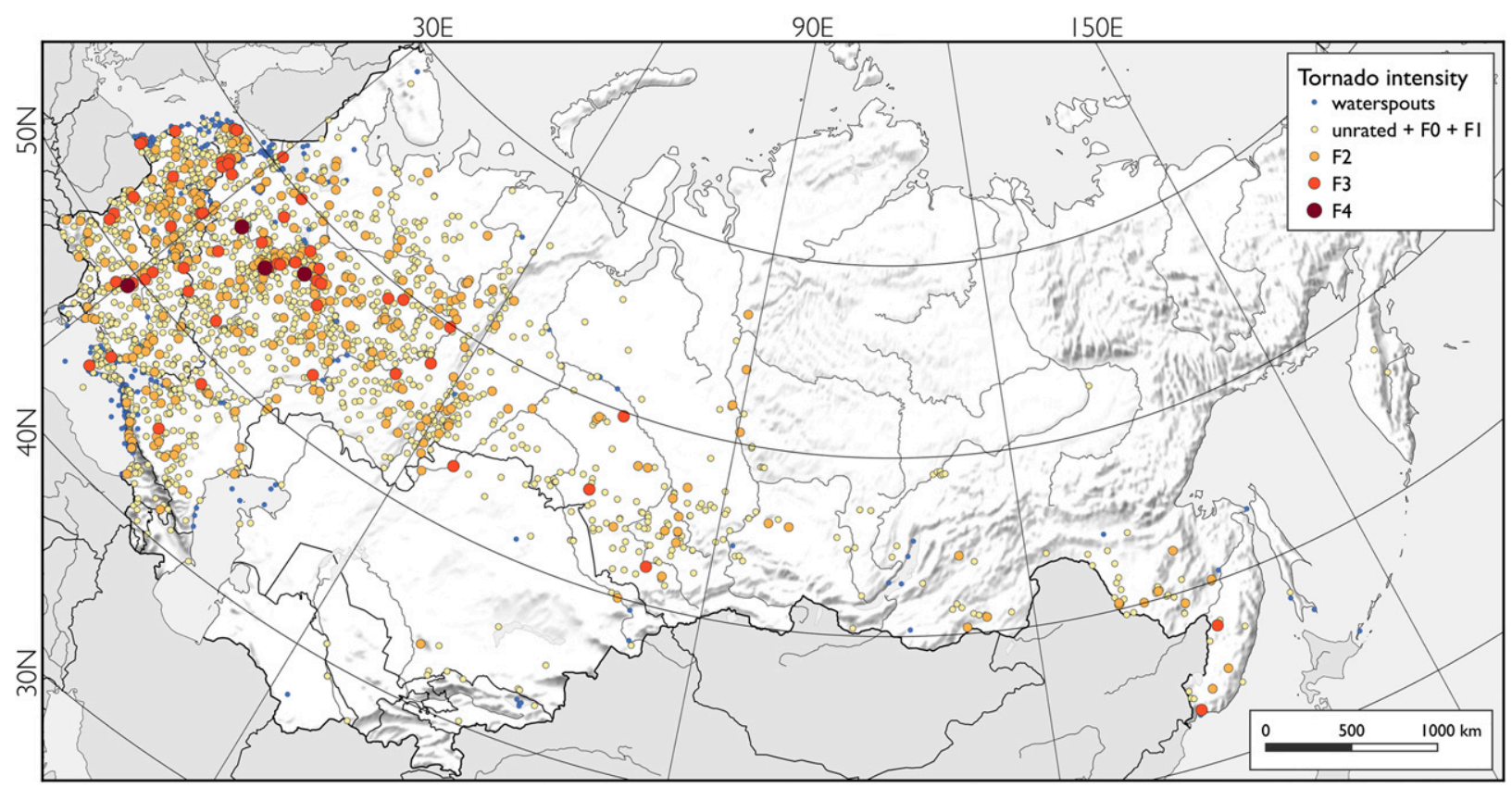

FIG. 4. Location of tornado cases in northern Eurasia with any event certainty for the entire period. Fujita intensity (FI) is shown with the color and size of the circles.

States and western Europe, respectively (Groenemeijer and Kühne 2014; Deng et al. 2016). Most waterspout cases are reported in the Black and Baltic Seas, and rarely over the Okhotsk Sea, inner lakes, and rivers. Although tornadoes occur in many regions of NE, they are nevertheless a rare meteorological event.

Overall event rarity and small sample size for tornadoes constrains detailed analysis of the spatial structure of tornado climatology (Doswell 2007b). However, several prominent features can be highlighted. The most pronounced feature is the overall dependence of tornado report density on population density, as in other regions (Anderson et al. 2007; Rauhala et al. 2012; Chen et al. 2017). Specifically, the high population density in the Moscow area is seen in the local tornado report maximum in the region (Fig. 5). In contrast, the low population

TABLE 3. Number of tornado cases for different countries in northern Eurasia. "All" are cases with all degrees of certainty; $H+M$ are cases with the high and medium degrees of certainty only.

\begin{tabular}{|c|c|c|c|c|c|c|c|c|c|c|}
\hline \multirow[b]{2}{*}{ Countries } & \multicolumn{2}{|c|}{$\begin{array}{l}\text { All tornadoes } \\
\text { over land }\end{array}$} & \multicolumn{2}{|c|}{$\begin{array}{l}\text { Significant } \\
\text { tornadoes over } \\
\text { land }(\geq \mathrm{F} 2)\end{array}$} & \multicolumn{2}{|c|}{$\begin{array}{c}\text { Intense } \\
\text { tornadoes over } \\
\text { land }(\geq \mathrm{F} 3)\end{array}$} & \multicolumn{2}{|c|}{ All waterspouts } & \multicolumn{2}{|c|}{$\begin{array}{l}\text { Land-falling } \\
\text { waterspouts }\end{array}$} \\
\hline & All & $H+M$ & All & $H+M$ & All & $H+M$ & All & $H+M$ & All & $H+M$ \\
\hline Armenia & 4 & 4 & 0 & 0 & 0 & 0 & 0 & 0 & 0 & 0 \\
\hline Azerbaijan & 4 & 1 & 0 & 0 & 0 & 0 & 0 & 0 & 0 & 0 \\
\hline Belarus & 159 & 108 & 38 & 38 & 4 & 4 & 13 & 5 & 0 & 0 \\
\hline Estonia & 68 & 58 & 16 & 15 & 6 & 6 & 24 & 24 & 0 & 0 \\
\hline Georgia & 23 & 10 & 5 & 2 & 0 & 0 & 24 & 19 & 7 & 3 \\
\hline Kazakhstan & 23 & 23 & 3 & 3 & 0 & 0 & 9 & 8 & 1 & 0 \\
\hline Kyrgyzstan & 4 & 3 & 0 & 0 & 0 & 0 & 4 & 3 & 0 & 0 \\
\hline Latvia & 52 & 34 & 4 & 1 & 1 & 0 & 23 & 20 & 2 & 1 \\
\hline Lithuania & 35 & 29 & 11 & 11 & 1 & 1 & 6 & 6 & 2 & 2 \\
\hline Moldova & 15 & 10 & 1 & 0 & 0 & 0 & 2 & 1 & 1 & 0 \\
\hline Russia & 1511 & 1121 & 255 & 223 & 43 & 39 & 559 & 512 & 100 & 88 \\
\hline Tadzhikistan & 0 & 0 & 0 & 0 & 0 & 0 & 0 & 0 & 0 & 0 \\
\hline Turkmenistan & 6 & 6 & 0 & 0 & 0 & 0 & 1 & 1 & 0 & 0 \\
\hline Ukraine & 285 & 215 & 43 & 36 & 7 & 6 & 29 & 25 & 6 & 6 \\
\hline Uzbekistan & 0 & 0 & 0 & 0 & 0 & 0 & 0 & 0 & 0 & 0 \\
\hline
\end{tabular}



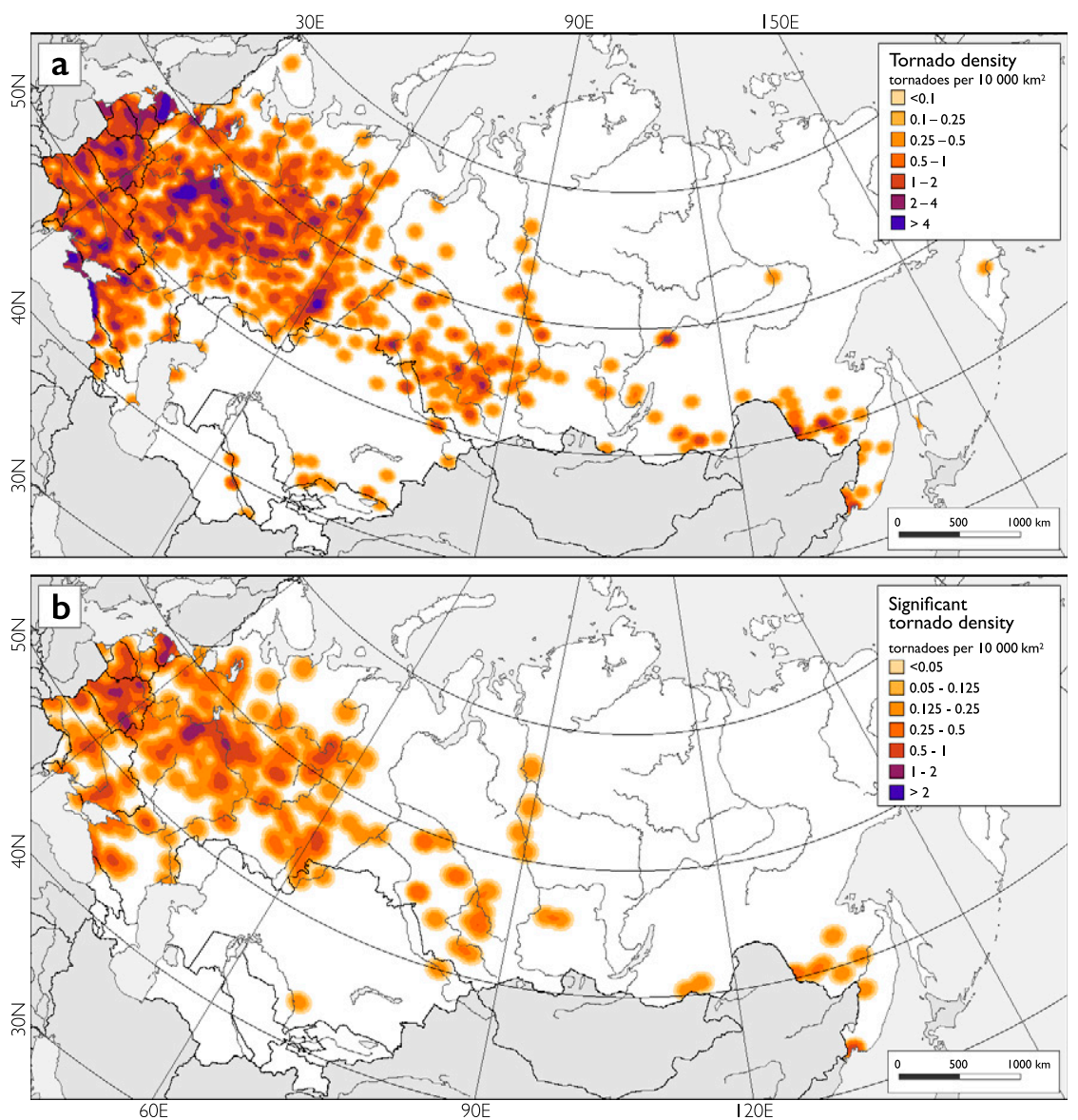

FIG. 5. Density of tornado cases per $10000 \mathrm{~km}^{2}$ for the 1900-2016 period for (a) all tornadoes over land and (b) significant tornadoes over land (with FI $\geq 2$ ). The density is calculated using Kernel density function (Silverman 1986) within (a) $20000 \mathrm{~km}^{2}$ and (b) $40000 \mathrm{~km}^{2}$ circled area around a point normalized by two and four, respectively. Only cases with high and medium certainty and with spatial accuracy less than $50 \mathrm{~km}$ are included. Note the different value ranges in (a) and (b).

density in the northeast regions of NE likely leads to an underestimation of tornado report numbers (Figs. 4, 5). A bias toward more intense tornadoes in populated areas is well pronounced in NE, similar to the United States (Doswell and Burgess 1988). Particularly, all four F4 tornadoes and the vast majority of F3 tornadoes (i.e., 53 out of 58) occur in the European part of $\mathrm{NE}$, and only five F3 tornadoes occur to the east of the Ural Mountains.

Satellite data on forest disturbances partly help to overcome the dependence on population, mostly for significant tornadoes (Fig. 5b) that are strong enough to affect forests. Yet, this leads to a dependence on the forest coverage. Particularly, using satellite data, we failed to find unreported tornado tracks in southern regions of $\mathrm{NE}$ that have few forests (Shikhov and Chernokulsky 2018). More frequent wildfires in southern Siberia and the Far East compared to the European part (Vivchar 2011) may mask out tornado tracks, which may lead to an underestimation of tornadoes in forests in the Asian part of NE. The local influence of forest coverage on tornado density is clear near the Yenisei River valley (Figs. 4, 5) where the percentage of forest area is higher compared to surrounding elevated regions (Fig. 2a). The presence of tornadoes in the valley, on both sides of the river, and the lack outside may point to a general underestimation of tornadoes in the region.

However, the topographic inhomogeneity itself may reduce the number of tornadoes (Karpman et al. 2013; Elsner et al. 2016). Particularly, a low number of tornadoes in Eastern Siberia and the north of Far East may be partly related to high surface-elevation roughness. That said this effect is likely smaller than the influence of low population density and forest coverage in the northern part of these regions (Fig. 2a). Another peculiar orographic effect is the local maxima of tornado reports in 
the lee of the South Ural Mountains. A number of studies demonstrated such intensification of convective processes in leeward regions (e.g., Giaiotti et al. 2007; Frame and Markowski 2006); however, we will not analyze the effect here in detail, leaving this for further research.

In addition to population density, forest cover, and orography, researcher engagement may influence the tornado reports. Comparing the number of tornado reports in Estonia, where much work had been done to collect reports (Tooming and Merilain 2004), with neighboring countries clearly demonstrates this effect (Table 3, Figs. 4, 5). Overall, the above examples point to a likely underestimation of the actual number of NE tornadoes.

Despite the aforementioned issues, the spatial distribution of the number of tornadoes generally corresponds to that for thunderstorm activity (Dai 2001a; Virts et al. 2013; Cecil et al. 2014) and favorable environments for tornado formation (Brooks et al. 2003; Tippett et al. 2015). The most northern tornado case from our database, specifically a waterspout, has latitude $68.8^{\circ} \mathrm{N}$. The most northern significant tornado with $\mathrm{F} 2$ intensity occurs at the latitude $66.78^{\circ} \mathrm{N}$, which generally corresponds to the northern boundary of tornado-prone environments (Brooks et al. 2003; Tippett et al. 2015) but is north of previous estimates (Voznyachuk 1954b; Snitkovskii 1987). The southward increase of lightning flash density (Virts et al. 2013; Cecil et al. 2014), thunderstorm occurrence (Dai 2001a), and days with tornado environments (Brooks et al. 2003) favors a greater density of tornadoes in the central and southern regions of $\mathrm{NE}$ between $50^{\circ}$ and $60^{\circ} \mathrm{N}$ latitudes. Large numbers of tornado reports near the Black Sea and low numbers over the dry desert-like area of central Asia correspond to high and low numbers of thunderstorm days (Dai 2001a) and number of lightning strokes (Virts et al. 2013; Cecil et al. 2014) in those regions. Both Brooks et al. (2003) and Tippett et al. (2015) likely underestimate the number of tornado environments over Western NE and overestimate this number over eastern NE. In particular, tornado reports provide little support for the existence of local maxima of tornado-prone environments in the southern Russian Far East (Brooks et al. 2003; Tippett et al. 2015), where high thunderstorm activity is revealed as well (Dai 2001a; Virts et al. 2013; Cecil et al. 2014). On one hand, the underestimate of the actual number of tornado reports in this low-populated region may take place. On the other hand, a relatively low tornado density has been shown not only over the southern Russian Far East but also in northeast China (Chen et al. 2017), which is a more densely populated region (GPWv4.11 2018). This regional mismatch between tornado-prone environments and actual tornado reports deserves further research. Such research should take into account the shortcomings of the existing estimates of Brooks et al. (2003) and Tippett et al. (2015), such as a limited period and poor resolution of the data used. It is beneficial to construct the climatology of tornado environments with the use of modern reanalyses (e.g., the ERA5 reanalysis) with better temporal, horizontal and vertical resolution.

\section{c. Annual cycle}

For estimating the annual and diurnal cycles, we used only cases with high and medium certainty and with time accuracy less than or equal to one week or one hour, respectively.

Tornadoes over land in NE have a distinct annual cycle, forming mostly in the warm period of a year (Fig. 6). In fact, $91.2 \%$ of all cases of tornadoes over land happen from May to August. None of the tornadoes occur in January. Significant and intense tornadoes form from February to October, and from May to September, respectively. June has the maximum of all tornadoes over land [i.e., 416 cases out of 1328 (31.3\%)], and of both significant and intense tornadoes [i.e., 90 out of $268(33.6 \%)$ and 14 out of $37(37.8 \%)$, respectively]. This is in contrast to Snitkovskii (1987), who found the maximum of NE tornadoes in July. The maximum in June comes from the use of new sources of information (e.g., satellite data) that shows many more tornadoes in June than in July (Shikhov and Chernokulsky 2018).

The annual cycle of tornado occurrence has regional variability. The European part of Russia (EPR) and Caucasus countries (Fig. 6d) experience a well-defined June maximum of tornadoes, while the western part of NE, Siberia, and central Asia have a comparably high number of tornadoes in both June and July. Based on the ESWD data, Groenemeijer and Kühne (2014) showed variability in the June and July maxima over the western part of NE and EPR and found a tornado maximum in July for most European countries. Heavy showers (Chernokulsky et al. 2018) and thunderstorms (Taszarek et al. 2019) over EPR have maximum in July, and only over the Caucasus does June show a maximum of showers (Chernokulsky et al. 2018). The Far East region has a different annual cycle of tornadoes compared to other regions (Fig. 6b). Tornadoes mostly form from July to September and they are rare in June. Convective precipitation here has a similar monsoondriven annual cycle with the maximum in July and August (Chernokulsky et al. 2018). Neighboring regions also experience a tornado maximum in the same period, particularly in July in northeastern China (Chen et al. 2017) and in September in Japan (Niino et al. 1997).

Waterspouts occur in all months with a minimum in late winter and a maximum in late summer when they 

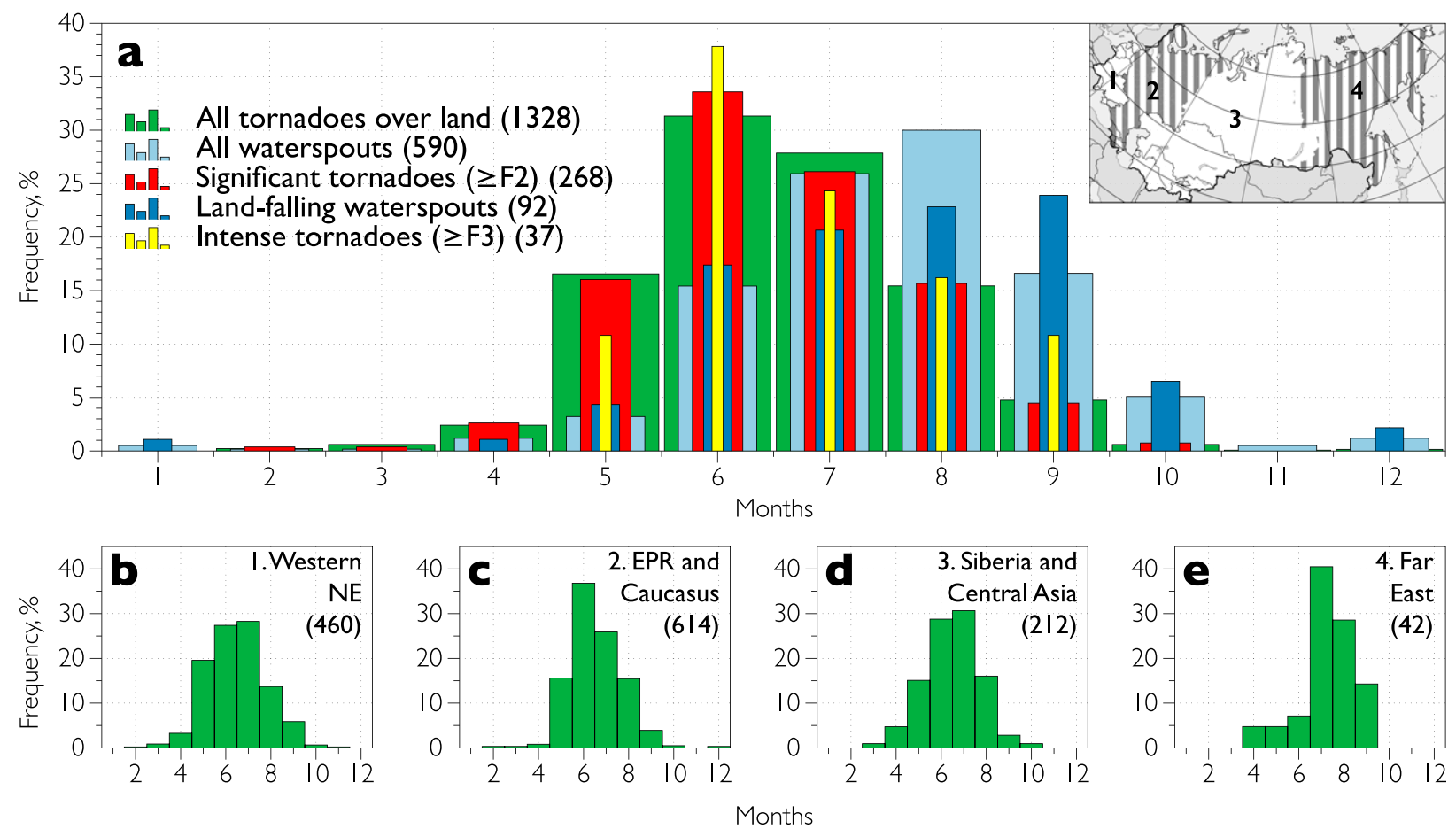

FIG. 6. The number of tornado cases in each month of the year. Numbers in brackets show the total number of cases used for each category. Tornadoes with different underlying surface and intensity are shown in (a); tornadoes over land with all intensities are shown in (b)-(e) for different regions (shown in inset). Only cases with high and medium certainty and time accuracy better than one week are included.

form above warm sea surface. Specifically, more than half of all waterspouts form in July and August. It is of note that many waterspout cases include more than one vortex (i.e., 1.46 on average), which is especially true in August (1.62). In almost every month, waterspouts occasionally make landfall, especially often from June to September. The month of September experiences the maximum of landfalling waterspouts, with almost a quarter of all landfalling waterspouts in that month. Miglietta and Matsangouras (2018) found a similar one-month shift between maxima of occurrence of all the waterspouts and of the landfalling ones for the Italian coast.

\section{d. Diurnal cycle}

Tornadoes over land in NE have a well-pronounced diurnal cycle with a minimum during the night and a maximum during the day (Fig. 7 ). Thus, $15 \%$ and $25 \%$ of all tornado cases and of intense tornado cases, respectively, are recorded from 1700 to $1800 \mathrm{LT}$, hereinafter local time (Fig. 7). Meanwhile, only one case is found from 0300 to 0400 LT. Atmospheric convection, indeed, follows the temperature diurnal cycle and develops in the afternoon after the temperature maximum. However, fewer of witnesses at night compared to daytime likely leads to an underestimate of the number of tornadoes that occurred during the night. For instance, the small sample size may be the reason for the lack of tornadoes occurring during the night over the Far East and Siberia (Figs. 7b,c). The diurnal cycle in Siberia (Fig. 7c) is of particular interest since it has the local minimum at 1800 LT between two maxima around 1600 and 2000 LT. This bimodality can be a sampling artifact or have natural causes and deserves further research.

Waterspouts are more evenly distributed throughout the day. They mostly occur from 0900 and 1300 LT, when 137 cases with 181 vortices were reported, out of the total number of 370 waterspouts and 494 vortices; however, they are also common earlier in the morning and in the afternoon. Waterspouts occur in a variety of synoptic situations (Sioutas and Keul 2007) but usually are associated with warm sea surface temperatures that sets conditions favorable for convective development during both day and nighttime. Therefore, the number of waterspouts occurring during nighttime is very likely underreported because of low visibility and few witnesses during the night - only two cases fall between midnight and 0400 LT.

These findings on the diurnal cycle are consistent with the other tornado- and convective-event-related studies. In particular, previous studies (e.g., Snitkovskii 1987; Niino et al. 1997; Rauhala et al. 2012; Groenemeijer and 

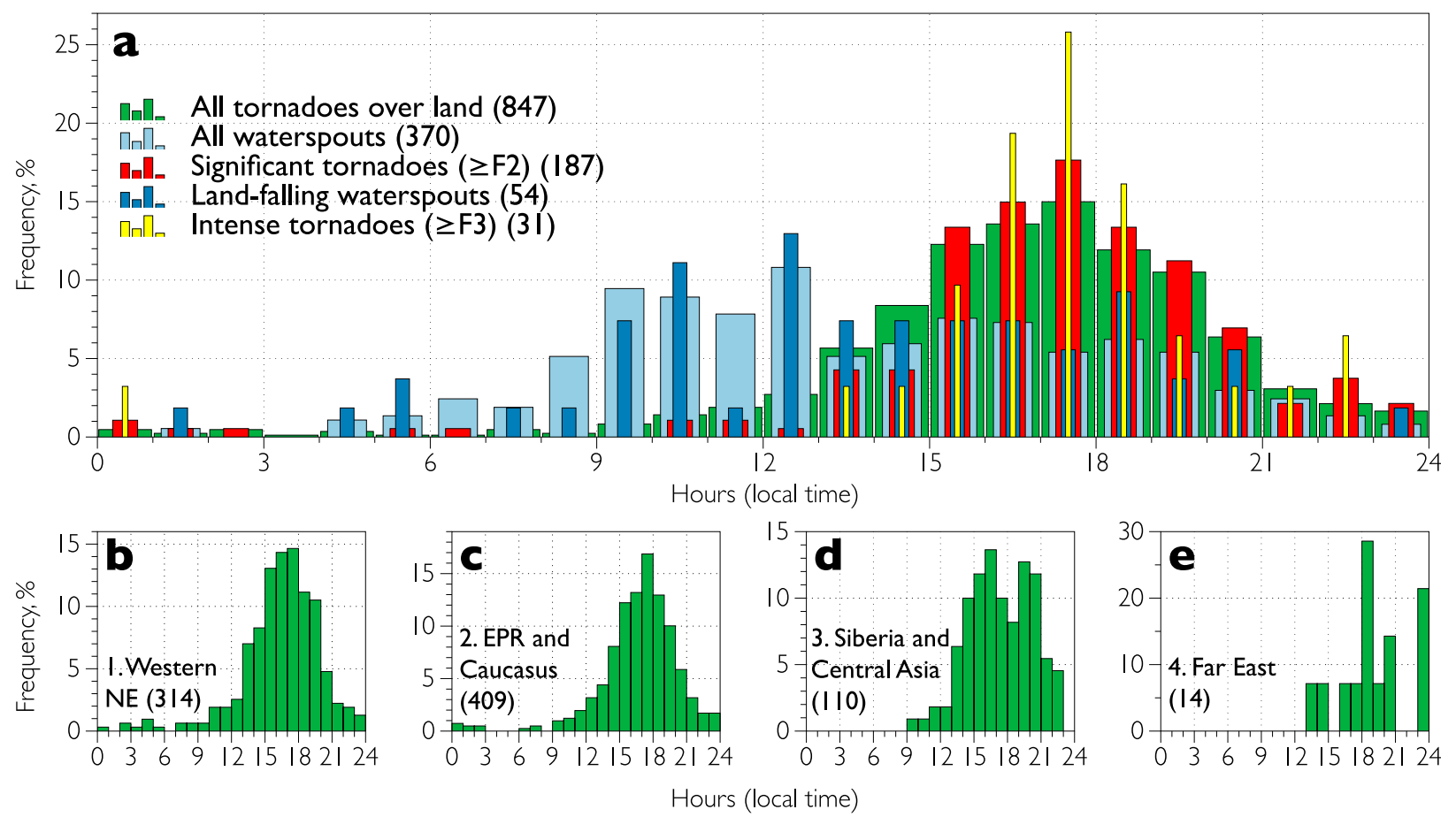

FIG. 7. The number of tornado cases in each hour of the day. Numbers in brackets show the total number of used cases for each category. (a) Tornadoes with different underlying surface and intensity; (b)-(e) tornadoes over land with all intensities for different regions (see inset in Fig. 6). Only cases with high and medium certainty and temporal accuracy $1 \mathrm{~h}$ and less are included.

Kühne 2014; Antonescu and Bell 2015; Taszarek and Brooks 2015; Chen et al. 2017) showed an afternoon maximum of tornadoes over land for NE and neighboring countries. Dai (2001b) determined the same time (1600-1800 LT) for the diurnal maximum of NE thunderstorms. The diurnal cycle of waterspouts, that is, flatter than for tornadoes over land, and with higher values before noon, is similar to that previously observed over the Baltic (Rauhala et al. 2012), Mediterranean (Miglietta and Matsangouras 2018), and Japan Seas (Niino et al. 1997). Despite the good agreement among different regions, some characteristics of the tornado diurnal cycle are uncertain. In particular, an apparent underreporting of nocturnal tornadoes, especially over water, prevents accurate estimates of the diurnal cycle amplitude.

\section{Tornado characteristics}

\section{a. Tornado intensity}

Most of tornadoes in NE are weak (Fig. 8, Table 2). Approximately $80 \%$ of all tornadoes over land have $\mathrm{F} 0$ or F1 intensity and $<3 \%$ have F3 or F4 intensity. No F5 tornadoes have been recorded. Groenemeijer and Kühne (2014) suggested that the tornado that hit
Ivanovo on 9 June 1984 might be F5; however, a more careful examination by Chernokulsky and Shikhov (2018) put it into high end of the F4 rating. The fraction of intense tornadoes $(\geq F 3)$ among all tornadoes is smaller than in Europe and the United States, but larger than for China (Fig. 8). In general, the distribution of NE tornado intensities can be reasonably well approximated with a Rayleigh distribution over peak wind speeds (Kurgansky 2000; Dotzek et al. 2005) except for the F0 rating, even if all unrated tornadoes fall into this category, as suggested in Grünwald and Brooks (2011) (Fig. 8).

The number of tornadoes with the F0 rating is likely underreported in NE. On one hand, the share of F0 in historical cases is small and increases in time with more modern reports. In particular, only three F0 cases out of 35 rated tornadoes with high and medium certainty were reported before 1900,18 out of 96 before 1950, while 329 out of 990 after 2000. On the other hand, F0 tornadoes are missed in satellite data (Shikhov and Chernokulsky 2018) and also can be missed in rural areas compared to stronger tornadoes (Anderson et al. 2007). Thus, the "true" tornado intensity distribution in NE should show an even greater portion of weak tornadoes than it does now. 


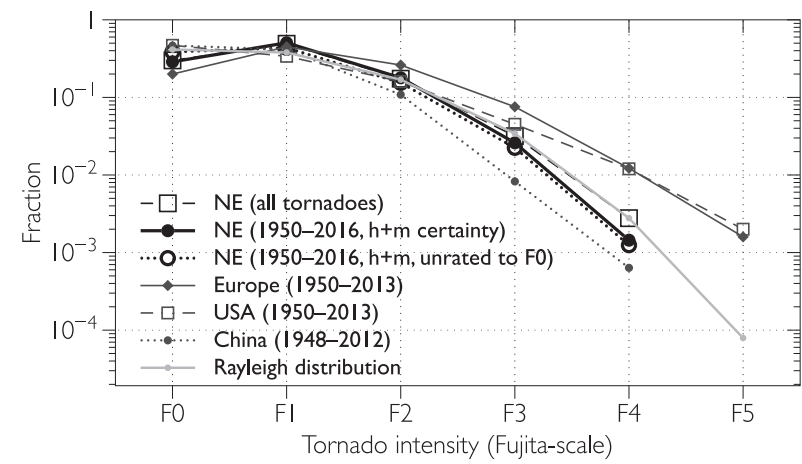

FIG. 8. Tornado Fujita intensity distribution in northern Eurasia (this study) for all cases for NE (black dashed line with open squares), for cases for the period 1950-2016 with the high and medium certainty (black thick solid line with closed circles), for cases for the period 1950-2016 with the high and medium certainty and with unrated tornadoes included into the F0 category (black dotted line with open circles). For comparison, distributions for other regions are shown. Data for Europe (gray solid line with closed diamonds) were adopted from Groenemeijer and Kühne (2014). Data for the United States (gray dashed line with open squares) were adopted from Groenemeijer and Kühne (2014). Data for China (gray dotted line with closed circles) were adopted from Chen et al. (2017). Light gray line shows the reference Rayleigh distribution of tornadoes on their intensity (peak wind speed class) calculated from data on the Fujita intensity of all NE tornadoes using the methodology by Dotzek et al. (2005).

\section{b. Tornado path geometrical characteristics}

Our database shows that half of all tornadoes have a pathlength greater than $6.7 \mathrm{~km}$, while maximum and mean width is greater than 250 and $120 \mathrm{~m}$, respectively (Figs. 9-11 ). Average values of tornado length and mean width (i.e., $12.7 \mathrm{~km}$ and $167 \mathrm{~m}$ ) in our database are noticeably higher than those for U.S. tornadoes, $7.1 \mathrm{~km}$ and $117 \mathrm{~m}$, respectively (Schaefer et al. 1986). The difference is likely due to sampling bias resulting from the lack of practice of tornado damage surveys in NE. Commonly, stronger tornado cases in NE have more information available on their path sizes. Specifically, $44 \%$ of $\mathrm{F} 3$ and $100 \%$ of F4 tornado reports in our database have information on pathlength and width, while $93 \%$ of $\mathrm{F} 0$ and $86 \%$ of $\mathrm{F} 1$ tornado reports lack this information. In addition, all satellite-derived tornadoes have path and length estimates, and these tornadoes inherently have intensity $\geq \mathrm{F} 1$ for being capable of damaging forest (Shikhov and Chernokulsky 2018).

In general, stronger $\mathrm{NE}$ tornadoes have longer and wider path (Figs. 9b, 10b, 11b), which is concordant with findings for tornadoes in Europe (Groenemeijer and Kühne 2014) and the United States (Schaefer et al. 1986; McCarthy 2003; Brooks 2004). Estimates of the geometric characteristics for each F-scale rating are presumably less biased. However, they are similar to

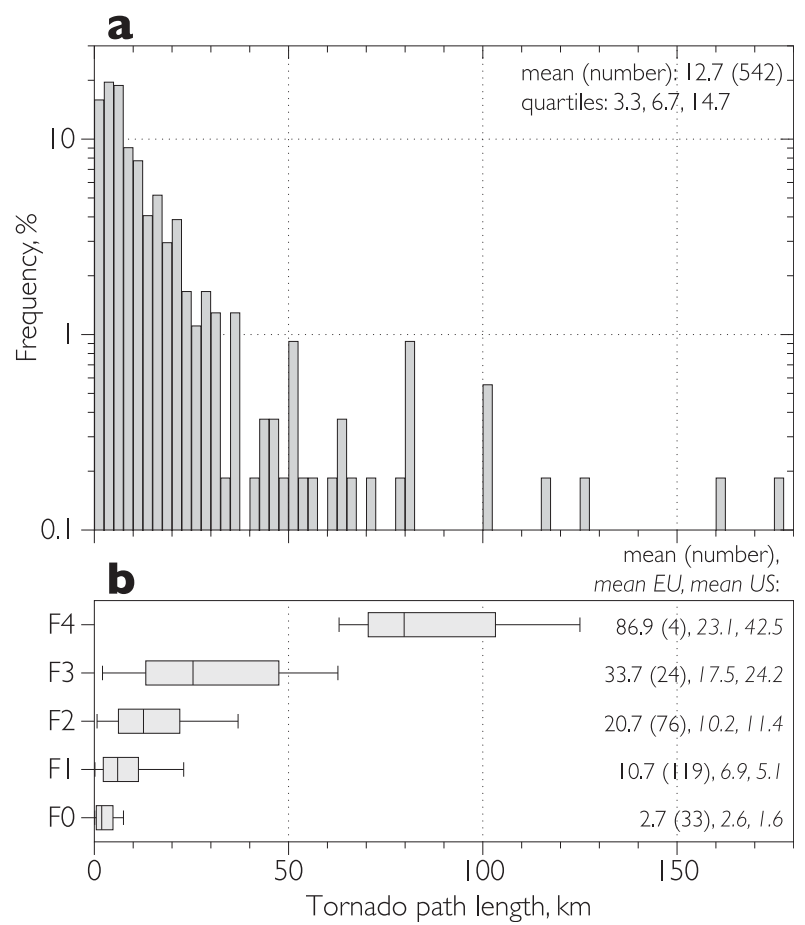

FIG. 9. Distribution of the pathlength of tornadoes with high and medium certainty only: (a) distribution of tornadoes of any intensity with known pathlength; the number of tornadoes used is shown in brackets; the mean and quartiles values are also shown. (b) Boxand-whisker plots for pathlength of tornadoes with the particular Fujita intensity-only for tornadoes with known intensity and pathlength; satellite-derived tornadoes with intensity obtained as length/width-determined probability estimate are excluded; the mean length and the number of tornadoes used (in brackets) are shown for each intensity rating; additionally, mean lengths of tornadoes over Europe and the United States, which were adopted from Groenemeijer and Kühne (2014), are shown in italics.

those for European and U.S. tornadoes only for the F0 rating, and generally larger for other ratings. This may come from both small sampling (e.g., for the F4 rating) and low accuracy of historical estimates of geometrical characteristics - especially for pathlength and maximum width.

The length and width of the longest and widest tornadoes in our database are likely overestimated. The widest tornado path in the database (i.e., $2500 \mathrm{~m}$ ) belongs to the F4 tornado near Toropets (Tver region) that occurred in 1625. However, this estimate is approximate-[i.e., the source stated that the width of destruction path was " $2-3$ versts" ( 1 verst being equal to $1.067 \mathrm{~km})]$. More recent and more reliable satellitebased estimates show that the maximum tornado path width in NE reaches $2100 \mathrm{~m}$, which is half the width of the widest tornado in the United States (Bluestein et al. 2015). The tornado with this width was observed in Kostroma region on 12 June 2010. The longest tornado path in NE (i.e., $175 \mathrm{~km}$ ) occurred in 1964 in the Kirov 


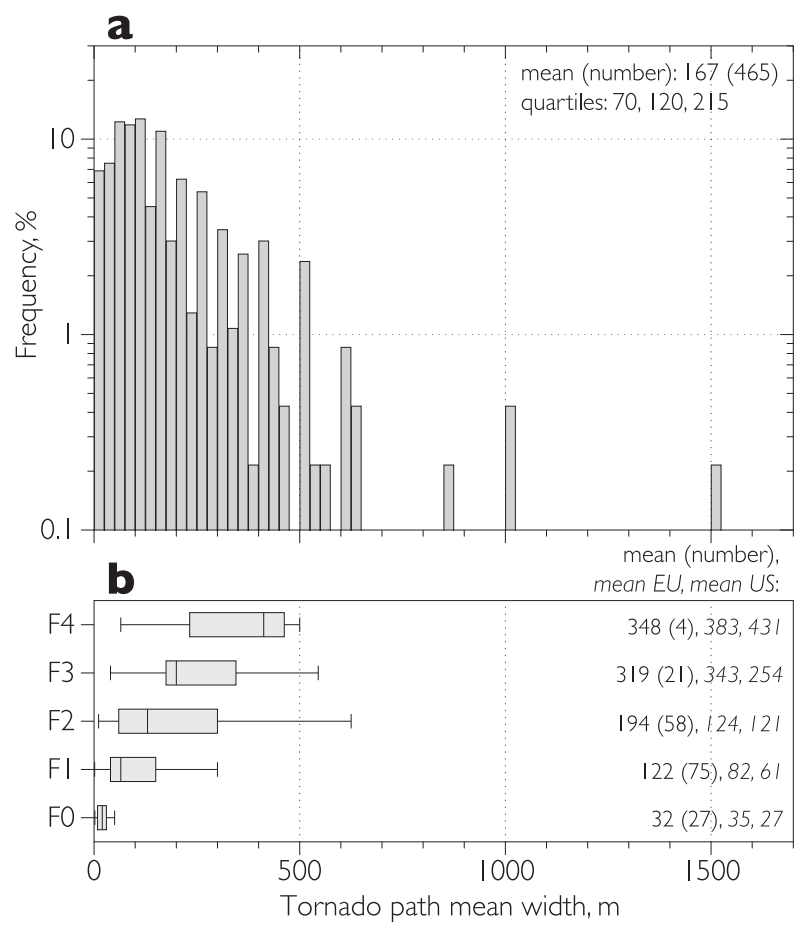

FIG. 10. As in Fig. 9, but for tornado path mean width.

Oblast and Udmurtiya Republic according to Snitkovskii (1987). However, no details were provided. In total, our database contains information on seven historical cases with tornado pathlengths $\geq 100 \mathrm{~km}$. All these long tracks are likely a series of short-path tornadoes. This possibility was illustrated for several historical cases in the United States (Doswell and Burgess 1988) and also for tornadoes of the Ivanovo outbreak in NE (Chernokulsky and Shikhov 2018), when the real longest path did not exceed $85 \mathrm{~km}$, which is half the value in the Snitkovskii database (Snitkovskii 1987). Using satellite data for the twenty-first century, Shikhov and Chernokulsky (2018) found tornadoes in forested areas with $80 \mathrm{~km}$ pathlengths. Consequently, the most reliable existing estimates of the longest and widest paths for reported tornadoes in NE are around $80-85$ and $2 \mathrm{~km}$, respectively.

\section{c. Tornado movement direction, duration, and rotation}

Tornadoes over NE form mostly in a warm sector of an extratropical cyclone, $50-100 \mathrm{~km}$ ahead of a cold front, or near an occlusion point (Snitkovskii 1987). They typically move eastward or northeastward, which determine the windrose pattern for tornado movement (Fig. 12). We found that half of all tornadoes over land with known direction of movement come from southwest (WSW, SW, and SSW), and only a few

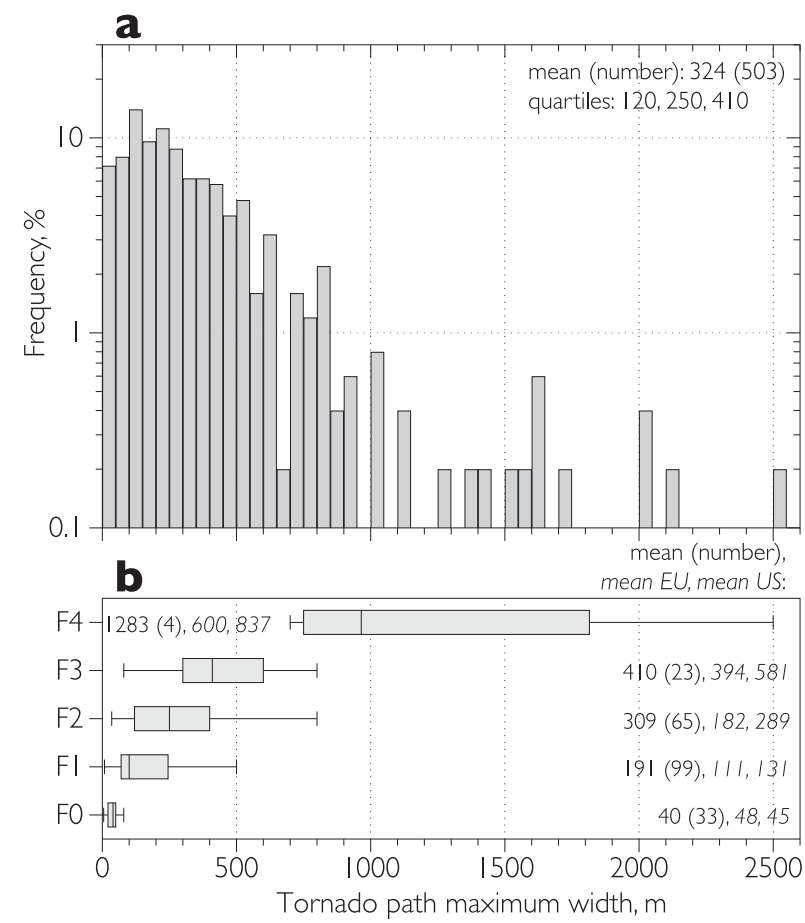

FIG. 11. As in Fig. 9, but for tornado path maximum width.

have the opposite direction. Given the smaller sample size, Snitkovskii (1987) reported $75 \%$ of all tornadoes to come from southwest, underestimating the number of tornadoes moving northward and eastward. These directions are characteristic for previously underreported tornadoes over Siberia and Ural. The coastline of the Black Sea, and of the Baltic Sea to a lesser degree, determines the prevailing directions of waterspout movement in NE-west, southwest, and southeast.

Tornadoes in NE are typically short lived. More than a half of all tornadoes last less than $10 \mathrm{~min}$ and only around $10 \%$ last more than $30 \mathrm{~min}$ (Table 4). Weak tornadoes have much shorter duration than significant ones. The mean lifetime of tornadoes over land and over water is somewhat equal. The longest-lived tornado (240 $\mathrm{min})$ and waterspout $(157 \mathrm{~min})$ both happened in the 1960s-70s. They were reported by Snitkovskii (1987), who likely overestimated their lifetime together with pathlength. More recently, the tornado with the longest lifetime (75 min) occurred in 2014 in Bashkiria (Chernokulsky et al. 2015). Our estimates of mean lifetime generally agree with those for tornadoes and waterspouts for other regions throughout the world (Table 4).

The overwhelming majority of tornadoes in NE have cyclonic rotation. Among 203 tornadoes with high and medium certainty and with known rotation direction, only five tornadoes had anticyclonic rotation, which 

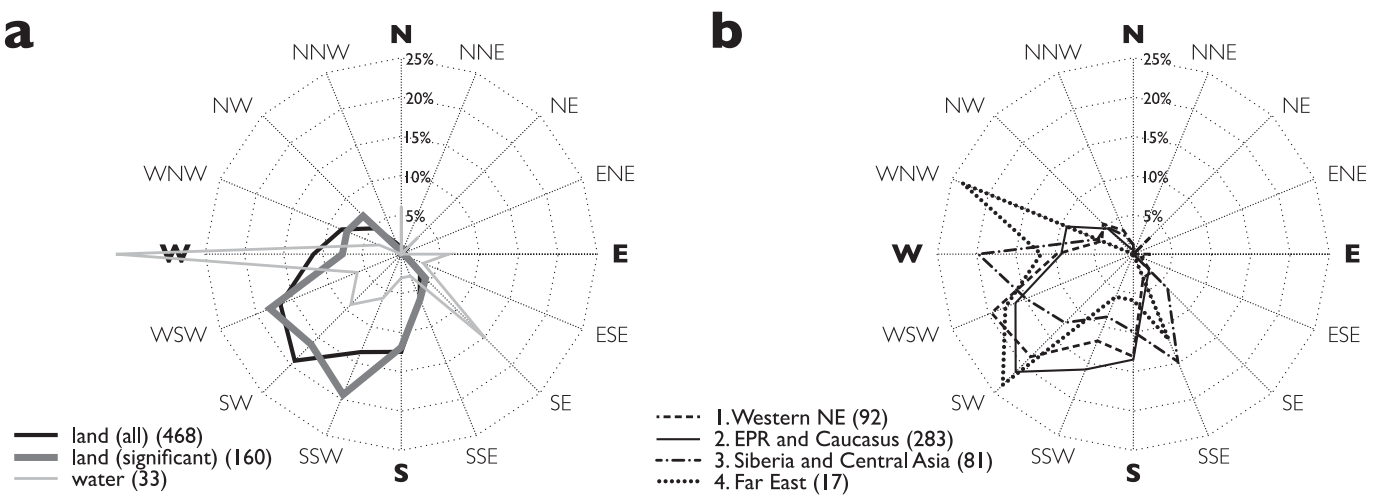

FIG. 12. The direction from which tornadoes originate, presented in 16 rhumbs. Numbers in brackets show the total number of cases used for each category. Tornadoes with different underlying surface and intensity are shown in (a), the landfalling waterspouts are included into the "water" category; tornadoes with the undefined surface are included into the "land" category. Tornadoes over land with all intensities for different regions (see inset in Fig. 6) are shown in (b) - note that five tornadoes belong to two regions. Only cases with high and medium certainty are included.

aligns with the rarity of anticyclonic tornadoes in other parts of the world (Fujita 1977).

\section{Tornado impact}

Tornadoes in NE have a noticeable environmental and socioeconomic impact. According to CCRC, some historical wind events resulted in thousands of casualties - particularly, in Rostov in 1408 and in Velikiy Novgorod in 1472. However, these cases have low certainty. From the nineteenth century, several tornado cases have led to dozens of casualties and substantial economic loss (see the Appendix). The exact number of casualties remains unknown because of the imperfect record keeping in the Russian Empire and the tendency to conceal information on catastrophes and related casualties in the USSR (see, e.g., Britkov and Sergeev 1998; Chernov and Sornette 2016; Gegel 2011; Piyp 2005). In particular, for the two most destructive tornado events of the twentieth century, the 1904
Moscow and the 1984 Ivanovo outbreaks, the reported minimum and maximum numbers of casualties and injures differ by two orders of magnitude (Table A1) (for additional details, see Nalivkin 1970; Groenemeijer and Kühne 2014; Chernokulsky and Shikhov 2018). Several intense tornadoes that resulted in substantial economic loss lack any information on casualties (Table A1). A large number of fatalities are characteristic for severe convective events on the Black Sea coast that are accompanied with landfalling waterspouts, heavy showers, and flash floods (e.g., the events of 8 August 2002, 1 August 1991, and 15 October 2010 resulted in 62,35 , and 26 fatalities, respectively). Flash floods play a major role in that high death toll, while the role of waterspouts in the flood enhancement is still unclear. Specifically, during the event of 2002, observers detected salty water in a rain gauge at the Novorossiysk meteorological station that came when waterspouts occurred that day. Relevant estimates can be made in further studies.

TABLE 4. Duration of tornadoes, with high and medium certainty only, that have relevant information for various categories.

\begin{tabular}{|c|c|c|c|c|c|c|}
\hline \multirow[b]{2}{*}{ Tornado type } & \multicolumn{4}{|c|}{$\begin{array}{c}\text { Tornado No. with specific } \\
\text { duration (\%) (for the entire period) }\end{array}$} & \multicolumn{2}{|c|}{$\begin{array}{l}\text { Mean }(\min ) \text { (No. of } \\
\text { tornadoes is shown in } \\
\text { brackets) }\end{array}$} \\
\hline & $\leq 5 \mathrm{~min}$ & $(5.15] \min$ & $(15.30] \min$ & $>30 \mathrm{~min}$ & The entire period & $2000-16$ \\
\hline All tornadoes over land & 31 & 23 & 37 & 9 & $16.1(219)$ & $11.8(95)$ \\
\hline Weak (F0, F1, unrated) & 37 & 22 & 34 & 7 & $13.4(160)$ & $9.7(73)$ \\
\hline Significant $(\geq \mathrm{F} 2)$ & 14 & 25 & 46 & 15 & $23.4(59)$ & $18.8(22)$ \\
\hline All tornadoes over water (with landfalling) & 27 & 26 & 36 & 11 & $17.1(74)$ & $12.0(43)$ \\
\hline \multicolumn{7}{|c|}{ Estimates for other regions } \\
\hline Tornadoes over land in Japan (Niino et al. 1997) & & & & & 12.0 & \\
\hline Waterspouts near Japan (Niino et al. 1997) & & & & & 14.0 & \\
\hline Waterspouts near Florida, the United States (Golden 1973) & & & & & 14.6 & \\
\hline Waterspouts near Italy (Miglietta and Matsangouras 2018) & & & & & 11.0 & \\
\hline
\end{tabular}



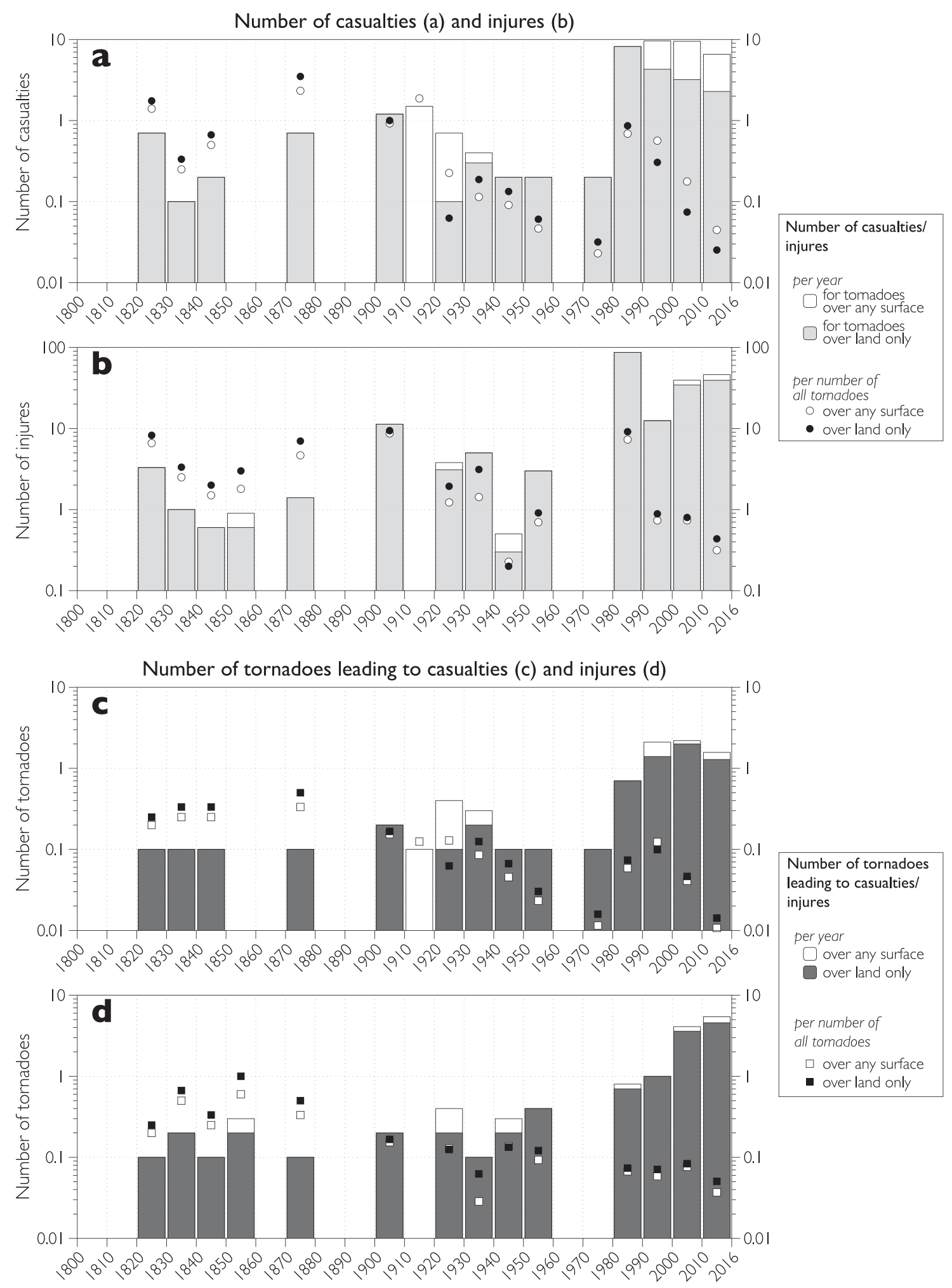

FIG. 13. Information on tornado victims: (a) the number of casualties per year for each period—decades from 1800 to 2009 (1800-09, 1810-91 etc.) and the last period for 2010-16-for tornadoes over land only (light gray bars) and for tornadoes over land or water including land-falling (white bars); the number of casualties per any tornado in each period over land only (black circles) and over land or water (white circles); (b) as in (a), but for the injured people number; (c) the number of killer tornadoes per year, counted for each period, over land only (dark gray bars) and over land or water (white bars); the ratio of killer tornadoes to all tornadoes over land only 
The high portion of weak tornadoes and a low population density mean a small percentage of tornadoes result in casualties (Fig. 13). In total, our database contains 114 cases with fatalities and 146 cases with injuries, including all degrees of certainty and, 79 and 121 cases for tornadoes with medium and high certainty (Table 2). After 1800, 78 tornado cases with high and medium certainty have resulted in at least 380 casualties and 119 tornado cases led to 2022 injuries. These numbers vary substantially from decade to decade (Fig. 13). As in other European countries (Groenemeijer and Kühne 2014), in the nineteenth and twentieth centuries the majority of fatalities came from intense tornadoes that occurred rather rarely, perhaps less than once per decade, while in the twenty-first century 1-2 tornadoes leading to casualties have occurred almost every year and yielded a rate of 2.9 fatalities and 36.3 injures per year. Although this rate is well below the United States with 79 deaths per year (Carbin and Guyer 2016), it is not negligible and illustrates the tornado threat to the society in NE.

\section{Conclusions}

We present a new database for tornadoes over northern Eurasia that covers 15 former USSR countries and fills the gap in the existing tornado climatologies for European countries (Antonescu et al. 2016). The database spans the period from the tenth century to 2016. The database is compiled using various sources, specifically, scientific case study papers and reviews, web news reports and scanned newspapers, national weather services' data including reports from routine meteorological observations, eyewitness reports, mainly from social networks that are subsequently checked by eyewitness interviewing, satellite data on tornado-induced forest disturbances, and data from the ESWD. The database contains 2879 tornado cases over land and water. It includes their time and place of occurrence, Fujita-scale intensity, duration, pathlength and width, movement and rotation direction, number of funnels per case, type of underlying surface, number of casualties, information on impact and primary sources, degree of event certainty. The database complements the paper as a separate CSV file in the online supplemental material.

The results show that tornadoes are ubiquitous for most regions of NE, especially for southern of Siberia and the Far East and for the entire European part of NE.
The density of all tornadoes reaches four cases per $10^{4} \mathrm{~km}^{2}$ in some regions, for significant tornadoes (intensity $\geq \mathrm{F} 2$ ), two cases per $10^{4} \mathrm{~km}^{2}$. The spatial distribution of tornado reports generally corresponds to the one for thunderstorms. However, issues with tornado detection lead to a higher tornado density in more populated and more forested regions with the most northern significant tornado beyond the Arctic Circle at the latitude $66.78^{\circ} \mathrm{N}$. Tornadoes over land in NE have a distinct annual cycle, forming mostly from May to August, when $>90 \%$ of all tornadoes were reported, with the maximum in June, when $>30 \%$ of all tornadoes and almost $40 \%$ of intense tornadoes occur. Although reported at all hours, tornadoes have a well-pronounced diurnal cycle with the daily maximum between 1700 and 1800 local time when $15 \%$ of all tornadoes and $25 \%$ of intense tornadoes occur. Waterspouts form in all months with a minimum in late winter and a maximum in late summer. They are more evenly distributed throughout the day and mostly occur between 0900 and 1300 local time. The dominant tornado movement direction is northeastward-around half of all tornadoes over land come from the southwest. The overwhelming majority of tornadoes in NE have cyclonic rotation.

Society substantially underestimates the threat of tornadoes in NE. The results presented here refute the common opinion on the extreme rarity of tornadoes in NE (Lyakhov 1987; Snitkovskii 1987). Almost every year, 1-3 intense tornadoes are reported, and the annual rate of significant tornadoes is $10 \pm 4$. Several intense tornadoes in NE had pathlengths up to $80-85 \mathrm{~km}$ and maximum widths up to $2 \mathrm{~km}$. The total annual number of all reported tornadoes over land is on the order of one hundred, and the annual number of reported tornado cases still shows a steady increase. Waterspouts occur at a rate of around 70-90 per annum and that rate is also increasing. Most of tornadoes are weak and prevalently short lived $-80 \%$ of all tornadoes are $\mathrm{F} 0 / \mathrm{F} 1$ and $<3 \%$ are $\mathrm{F} 3 / \mathrm{F} 4 ; 50 \%$ of tornadoes last $<10 \mathrm{~min}$. Despite this, tornadoes have a noticeable environmental and socioeconomic impact. From the beginning of the twenty-first century, 1-2 tornadoes every year were associated with casualties with 2.9 fatalities and 36.3 injures per year. More rarely, intense tornadoes that strike densely populated areas result in a greater number of casualties and significant economic loss-up to $0.02 \%$ of the annual GDP.

(black squares) and over land or water (white squares); (d) as in (c), but for the number of tornadoes causing injures. Only cases with high and medium certainty are included. The least number of casualties/injures is utilized when several estimates for one tornado case are presented. No fatalities are assumed for the cases where no data are available. Note the logarithmic ordinate scales in all plots. 


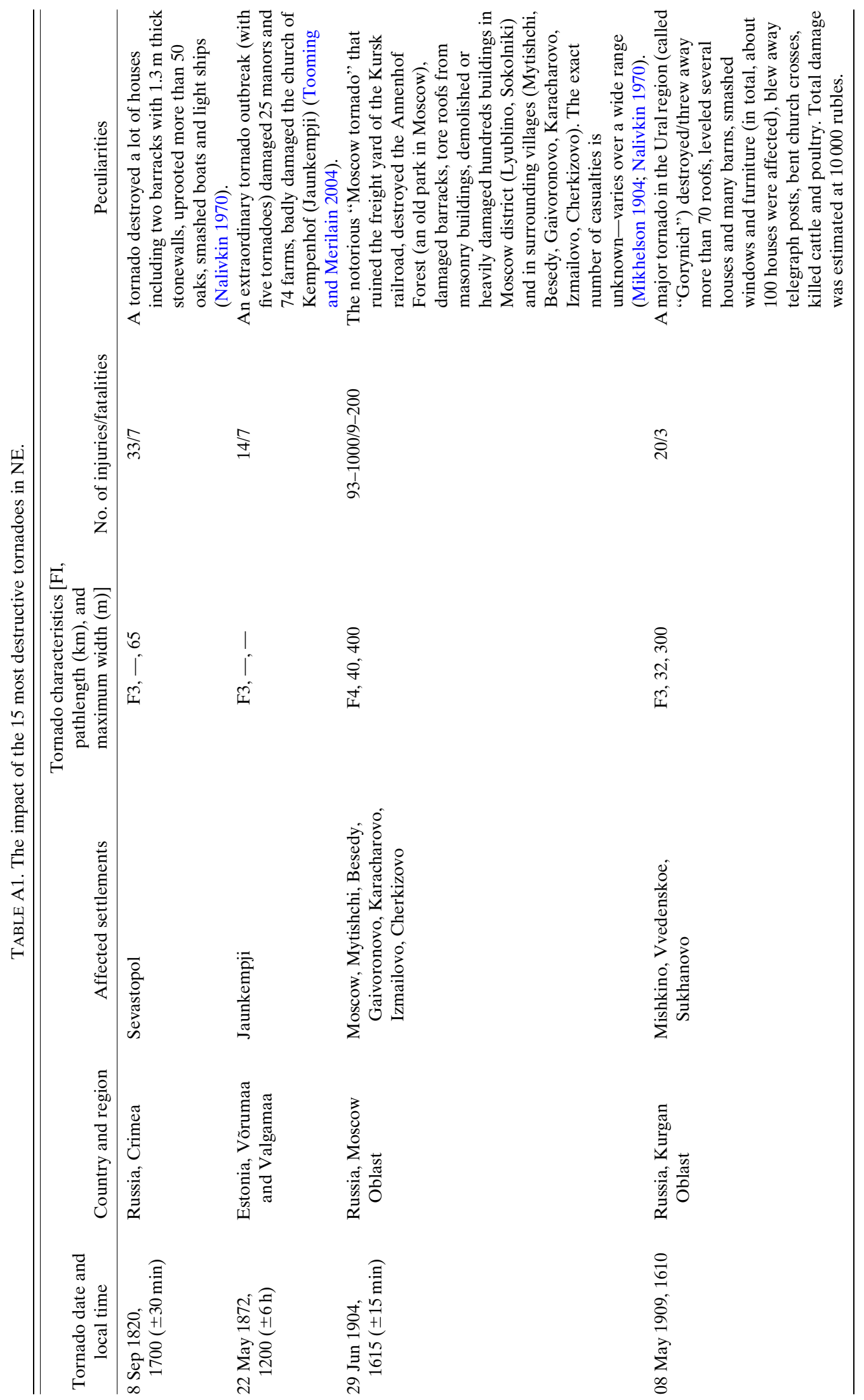




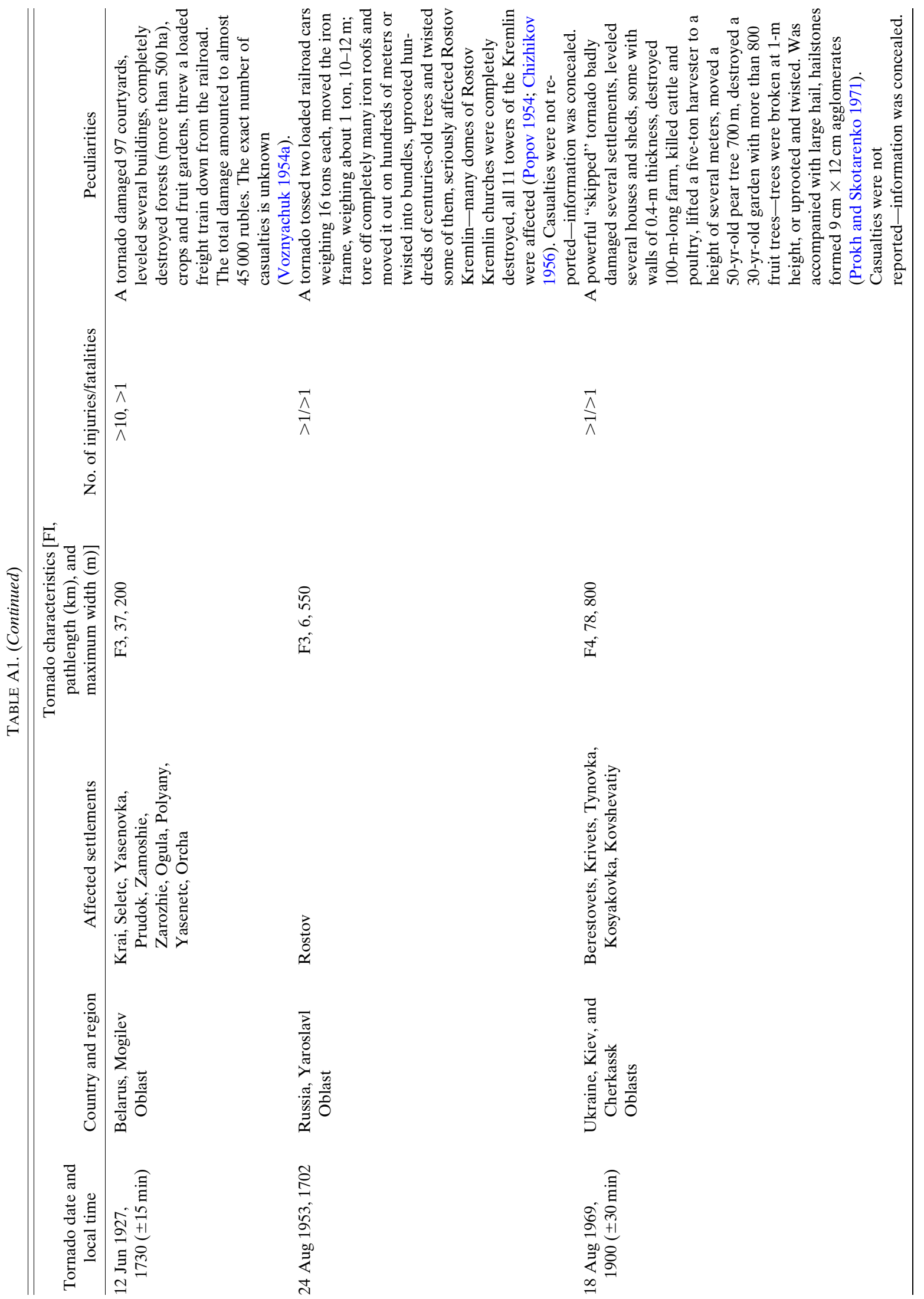




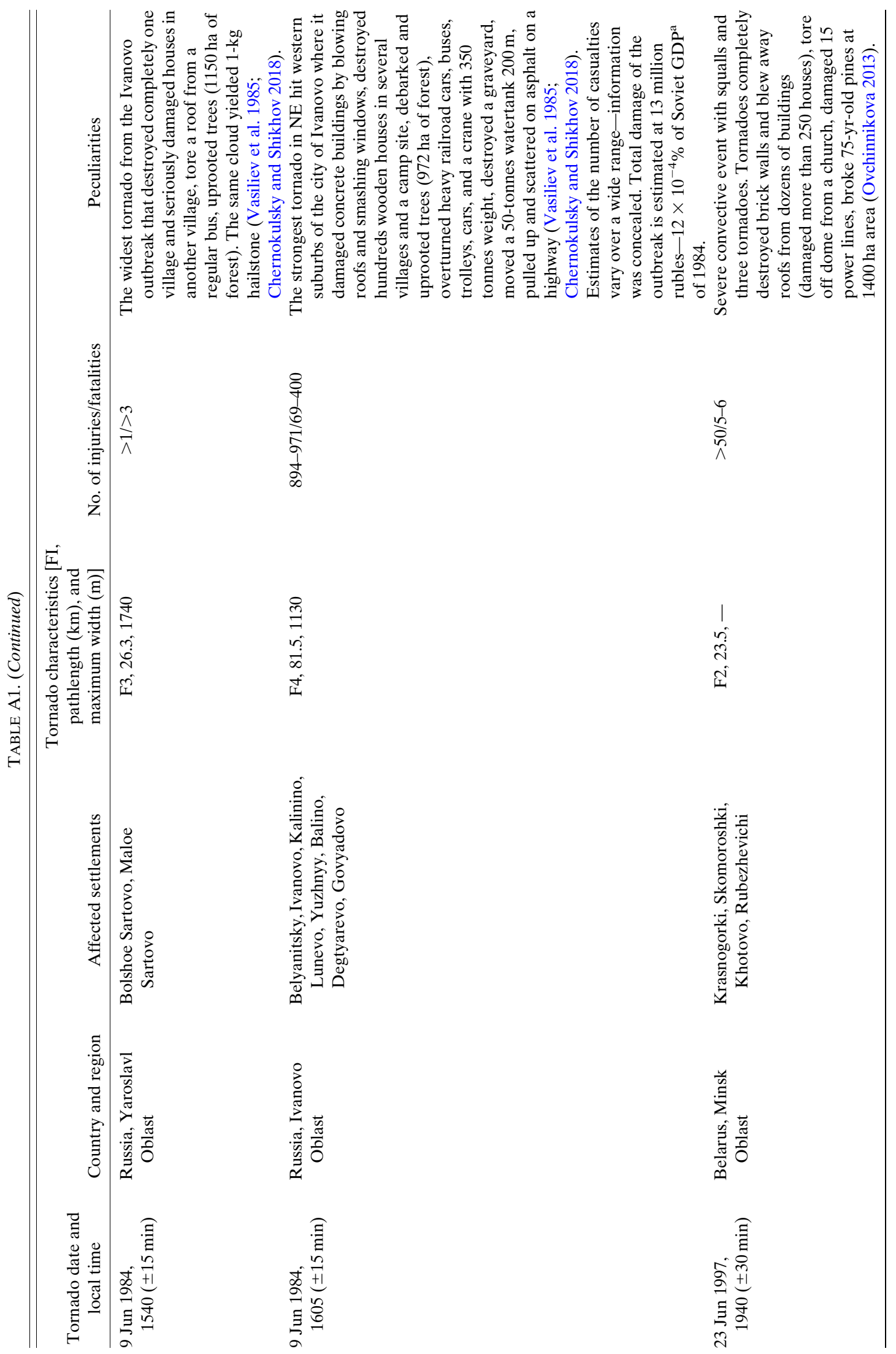




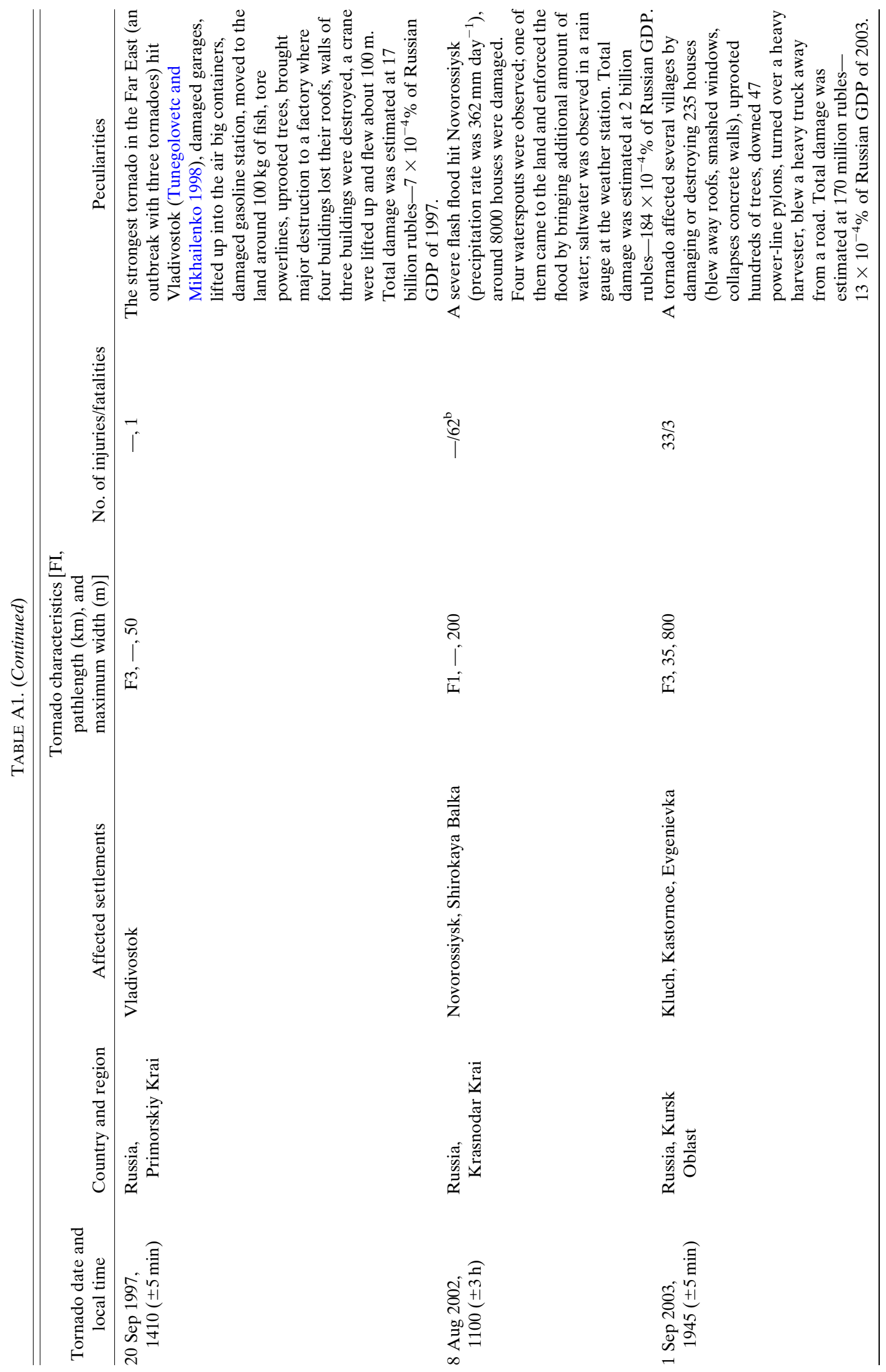




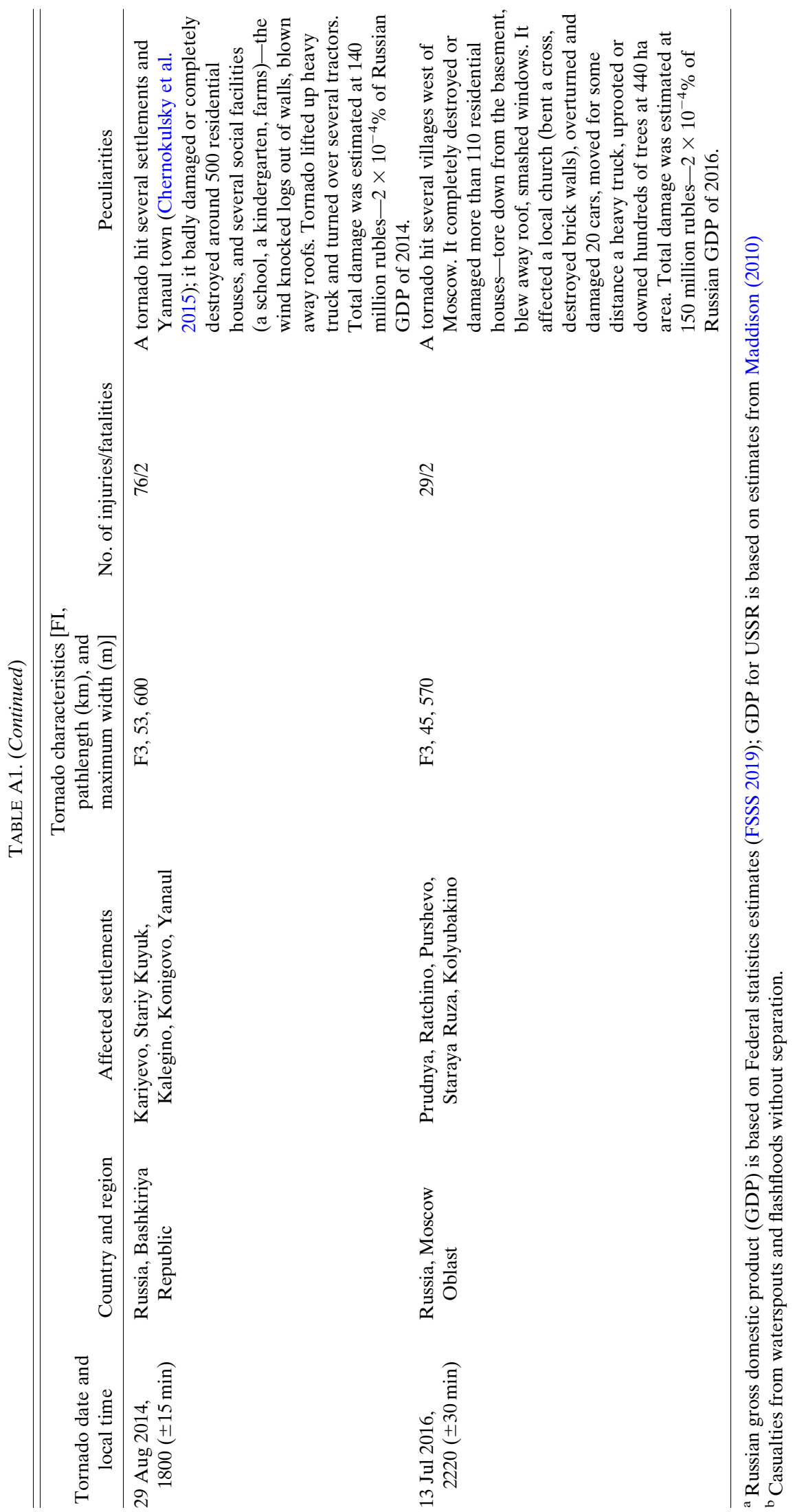


The results on the annual number of tornadoes and related socioeconomic impact unambiguously point to the need for systematic assessments and the forecasting of tornadoes by national weather services in NE. This is especially important under climate change conditions favoring an increase of risk of tornado formation in NE in the twenty-first century (Chernokulsky et al. 2017). The database is still incomplete, especially for weak tornadoes that are obviously underreported. Characteristics of tornadoes should be further refined: the intensity estimates will benefit from validating of $\mathrm{F}$ scale (or $\mathrm{EF}$ scale) against local construction practices and engineering codes (e.g., as performed in Japan) (Okada et al. 2015) and suggested for other regions (ESSL 2018). An estimate of the true climatology of tornadoes may be obtained by combining tornado reports and tornado environments (e.g., Brooks and Doswell 2001; Tippett et al. 2016), but solutions are needed for issues such as the apparent mismatch between a low number of tornado reports and a relatively high number of tornado prone days over the Far East. The climatology of tornado environments should be constructed using modern reanalyses (e.g., the ERA5 reanalysis) with high resolution. Weather radar observations should be actively involved in the diagnosis of tornadoes, for instance to reveal their mesocyclonic/nonmesocyclonic nature, as well as in tornado forecasting. The implementation of real-time forecasts and systematic assessments of tornadoes could lead to the reduction of risks associated with these hazardous meteorological phenomena in the future.

Acknowledgments. We appreciate Dr. Mateusz Taszarek, Dr. Pieter Groenemeijer, and the anonymous reviewer for their valuable comments that helped greatly to improve the article content and readability. We are also grateful to the Eastview Company for providing scanned newspapers, to all researchers whose studies on tornadoes in Northern Eurasia were used, and, finally, to all eyewitnesses who reported information on observed tornadoes. The study was supported by the Russian Science Foundation (Project 18-77-10076). The additional support for database collection was provided by the Russian Foundation for Basic Research (Projects 17-29-05098, 17-05-01097, and 19-0500046) and RAS Program "Climate Change: Causes, Risks, Consequences, Problems of Adaptation and Regulation."

\section{APPENDIX}

\section{The Most Destructive Tornadoes in Northern Eurasia}

The appendix provides information on the impact of the 15 most destructive tornadoes in NE from the beginning of the nineteenth century (Table A1). Events with only high certainty are presented.

\section{REFERENCES}

Anderson, C. J., C. K. Wikle, Q. Zhou, and J. A. Royle, 2007: Population influences on tornado reports in the United States. Wea. Forecasting, 22, 571-579, https://doi.org/10.1175/WAF997.1.

Antonescu, B., and A. Bell, 2015: Tornadoes in Romania. Mon. Wea. Rev., 143, 689-701, https://doi.org/10.1175/MWR-D-14-00181.1.

, D. M. Schultz, F. Lomas, and T. Kühne, 2016: Tornadoes in Europe: Synthesis of the observational datasets. Mon. Wea. Rev., 144, 2445-2480, https://doi.org/10.1175/MWR-D-15-0298.1.

,$- \ldots$, A. Holzer, and P. Groenemeijer, 2017: Tornadoes in Europe: An underestimated threat. Bull. Amer. Meteor. Soc., 98, 713-728, https://doi.org/10.1175/BAMS-D-16-0171.1.

Arino, O., J. J. Ramos Perez, V. Kalogirou, S. Bontemps, P. Defourny, and E. Van Bogaert, 2012: Global Land Cover Map for 2009 (GlobCover 2009). European Space Agency (ESA) and Université catholique de Louvain (UCL), PANGAEA, accessed 14 November 2018, https://doi.org/ 10.1594/PANGAEA.787668.

Bluestein, H. B., J. C. Snyder, and J. B. Houser, 2015: A multiscale overview of the El Reno, Oklahoma, tornadic supercell of 31 May 2013. Wea. Forecasting, 30, 525-552, https://doi.org/ 10.1175/WAF-D-14-00152.1.

Britkov, V., and G. Sergeev, 1998: Risk management: Role of social factors in major industrial accidents. Saf. Sci., 30, 173-181, https://doi.org/10.1016/S0925-7535(98)00046-0.

Brooks, H. E., 2004: On the relationship of tornado path length and width to intensity. Wea. Forecasting, 19, 310-319, https://doi.org/10.1175/1520-0434(2004)019<0310:OTROTP> 2.0.CO;2.

— , and C. A. Doswell III, 2001: Some aspects of the international climatology of tornadoes by damage classification. Atmos. Res., 56, 191-201, https://doi.org/10.1016/S0169-8095(00) 00098-3.

- — - and J. Cooper, 1994: On the environments of tornadic and nontornadic mesocyclones. Wea. Forecasting, 9, 606-618, https://doi.org/10.1175/1520-0434(1994)009<0606:OTEOTA $>$ 2.0.CO;2.

, J. W. Lee, and J. P. Craven, 2003: The spatial distribution of severe thunderstorm and tornado environments from global reanalysis data. Atmos. Res., 67-68, 73-94, https://doi.org/ 10.1016/S0169-8095(03)00045-0.

_ G. W. Carbin, and P. T. Marsh, 2014: Increased variability of tornado occurrence in the United States. Science, 346, 349352, https://doi.org/10.1126/science.1257460.

Bulygina, O. N., V. M. Veselov, V. N. Razuvaev, and T. M. Aleksandrova, 2014: Three-hourly meteorological observations from Russian meteorological stations. Accessed 9 September 2018, http://meteo.ru/english/climate/descrip12.htm.

Bunting, W. F., and B. E. Smith, 1993: A guide for conducting convective windstorm surveys. NOAA Tech. Memo. NWSSR-146, 44 pp.

Carbin, G., and J. Guyer, 2016: The 2015 tornado season: In like a lamb, out like a lion. Weatherwise, 69, 43-49, https://doi.org/ 10.1080/00431672.2016.1159490.

CCRC, 1841: Complete Collection of Russian Chronicles (43 volumes) (in Russian). The Highest Command of the Archaeographic Commission, Saint-Petersbourg, accessed 9 September 2018, http://psrl.csu.ru/indexs/index_tom.shtml. 
Cecil, D. J., D. E. Buechler, and R. J. Blakeslee, 2014: Gridded lightning climatology from TRMM-LIS and OTD: Dataset description. Atmos. Res., 135-136, 404-414, https://doi.org/ 10.1016/j.atmosres.2012.06.028.

Chen, J., and Coauthors, 2017: Tornado climatology of China. Int. J. Climatol., 38, 2478-2489, https://doi.org/10.1002/joc.5369.

Chernokulsky, A. V., and A. Shikhov, 2018: 1984 Ivanovo tornado outbreak: Determination of actual tornado tracks with satellite data. Atmos. Res., 207, 111-121, https://doi.org/10.1016/ j.atmosres.2018.02.011.

, M. V. Kurgansky, D. I. Zakharchenko, and I. I. Mokhov, 2015: Genesis environments and characteristics of the severe tornado in the South Urals on August 29, 2014. Russ. Meteor. Hydrol., 40, 794-799, https://doi.org/10.3103/S1068373915120031.

,-- , and I. I. Mokhov, 2017: Analysis of changes in tornadogenesis conditions over Northern Eurasia based on a simple index of atmospheric convective instability. Dokl. Earth Sci., 477, 1504-1509, https://doi.org/10.1134/S1028334X17120236.

— , F. A. Kozlov, O. G. Zolina, O. N. Bulygina, and V. A. Semenov, 2018: Climatology of precipitation of different genesis in Northern Eurasia. Russ. Meteor. Hydrol., 43, 425435, https://doi.org/10.3103/S1068373918070014.

Chernov, D., and D. Sornette, 2016: Man-made Catastrophes and Risk Information Concealment: Case Studies of Major Disasters and Human Fallibility. Springer, 342 pp., https://doi.org/10.1007/ 978-3-319-24301-6.

Chizhikov, N. V., 1956: Tornadoes in the Yaroslavl region in the summer 1953 (in Russian). Reg. Notes Yaroslavl Mus., 1, 67-85.

Dai, A., 2001a: Global precipitation and thunderstorm frequencies. Part I: Seasonal and interannual variations. J. Climate, 14, 1092-1111, https://doi.org/10.1175/1520-0442(2001)014<1092: GPATFP $>2.0 . \mathrm{CO} ; 2$.

_ 2001b: Global precipitation and thunderstorm frequencies. Part II: Diurnal variations. J. Climate, 14, 1112-1128, https://doi.org/ 10.1175/1520-0442(2001)014<1112:GPATFP $>2.0 . C O ; 2$.

Dee, D. P., and Coauthors, 2011: The ERA-Interim reanalysis: Configuration and performance of the data assimilation system. Quart. J. Roy. Meteor. Soc., 137, 553-597, https://doi.org/ 10.1002/qj.828.

Deng, Y., B. Wallace, D. Maassen, and J. Werner, 2016: A few GIS clarifications on tornado density mapping. J. Appl. Meteor. Climatol., 55, 283-296, https://doi.org/10.1175/JAMC-D-15-0141.1.

Doswell, C. A., III, 2003: A Guide to F-Scale Damage Assessment. U.S. Department of Commerce, $101 \mathrm{pp}$.

2007a: Historical overview of severe convective storms research. Electron. J. Severe Storms Meteor., 2 (1), https:// ejssm.org/ojs/index.php/ejssm/article/viewArticle/13/15.

- 2007b: Small sample size and data quality issues illustrated using tornado occurrence data. Electron. J. Severe Storms Meteor., 2 (5), https://ejssm.org/ojs/index.php/ejssm/article/ viewArticle/26/27.

— climatology. Mon. Wea. Rev., 116, 495-501, https://doi.org/ 10.1175/1520-0493(1988)116<0495:OSIOUS $>2.0 . C O ; 2$

- H. E. Brooks, and N. Dotzek, 2009: On the implementation of the enhanced Fujita scale in the USA. Atmos. Res., 93, 554563, https://doi.org/10.1016/j.atmosres.2008.11.003.

Dotzek, N., 2003: An updated estimate of tornado occurrence in Europe. Atmos. Res., 67-68, 153-161, https://doi.org/10.1016/ S0169-8095(03)00049-8.

, 2009: Derivation of physically motivated wind speed scales. Atmos. Res., 93, 564-574, https://doi.org/10.1016/ j.atmosres.2008.10.015.
— M. V. Kurgansky, J. Grieser, B. Feuerstein, and P. Névir, 2005: Observational evidence for exponential tornado intensity distributions over specific kinetic energy. Geophys. Res. Lett., 32, L24813, https://doi.org/10.1029/2005GL024583.

_ , P. Groenemeijer, B. Feuerstein, and A. M. Holzer, 2009: Overview of ESSL's severe convective storms research using the European Severe Weather Database ESWD. Atmos. Res., 93, 575-586, https://doi.org/10.1016/j.atmosres.2008.10.020.

Dyaduchenko, V., Y. B. Pavlyukov, and I. Vylegzhanin, 2014: Doppler weather radars in Russia (in Russian). Sci. Russ., 1, 23-27.

Dyer, R. C., 1988: Remote sensing identification of tornado tracks in Argentina, Brazil, and Paraguay. Photogramm. Eng. Remote Sens., 54, 1429-1435.

Edwards, R., J. G. LaDue, J. T. Ferree, K. Scharfenberg, C. Maier, and W. L. Coulbourne, 2013: Tornado intensity estimation: Past, present, and future. Bull. Amer. Meteor. Soc., 94, 641653, https://doi.org/10.1175/BAMS-D-11-00006.1.

Elsner, J. B., and Coauthors, 2016: The relationship between elevation roughness and tornado activity: A spatial statistical model fit to data from the central Great Plains. J. Appl. Meteor. Climatol., 55, 849-859, https://doi.org/10.1175/JAMCD-15-0225.1.

ESSL, 2018: International Fujita scale. Accessed 3 November 2019, https://www.essl.org/cms/international-fujita-scale/.

Feuerstein, B., P. Groenemeijer, E. Dirksen, M. Hubrig, A. M. Holzer, and N. Dotzek, 2011: Towards an improved wind speed scale and damage description adapted for Central Europe. Atmos. Res., 100, 547-564, https://doi.org/10.1016/ j.atmosres.2010.12.026.

Finch, J., and D. Bikos, 2012: Russian tornado outbreak of 9 June 1984. Electron. J. Severe Storms Meteor., 7 (4), http:// www.ejssm.org/ojs/index.php/ejssm/article/viewArticle/98.

Frame, J., and P. Markowski, 2006: The interaction of simulated squall lines with idealized mountain ridges. Mon. Wea. Rev., 134, 1919-1941, https://doi.org/10.1175/MWR3157.1.

FSSS, 2019: Russian Federation Federal State Statistics Service-Production of GDP. Accessed 14 November 2018, http://www.gks.ru/wps/wcm/connect/rosstat_main/rosstat/en/ figures/domestic/.

Fujita, T. T., 1977: Anticyclonic tornadoes. Weatherwise, 30, 51-64, https://doi.org/10.1080/00431672.1977.9931796.

- 1981: Tornadoes and downbursts in the context of generalized planetary scales. J. Atmos. Sci., 38, 1511-1534, https://doi.org/ 10.1175/1520-0469(1981)038<1511:TADITC $>2.0 . C O ; 2$.

Gaigerov, S. S., 1939: Six tornadoes in central regions of the Soviet European part and their synoptic conditions (in Russian). Sov. Meteor. Gidrol., 5, 44-54.

Gegel, I.V., 2011: Authorities and the press facing the accident on Chernobyl nuclear station (in Russian). Mediaskop, 2, 37-47.

Giaiotti, D. B., M. Giovannoni, A. Pucillo, and F. Stel, 2007: The climatology of tornadoes and waterspouts in Italy. Atmos. Res., 83, 534-541, https://doi.org/10.1016/j.atmosres.2005.10.020.

Golden, J. H., 1973: Some statistical aspects of waterspout formation. Weatherwise, 26, 108-117, https://doi.org/10.1080/ 00431672.1973 .9931643$.

Goliger, A. M., and R. V. Milford, 1998: A review of worldwide occurrence of tornadoes. J. Wind Eng. Ind. Aerodyn., 74-76, 111-121, https://doi.org/10.1016/S0167-6105(98)00009-9.

GPWv4.11, 2018: Gridded Population of the World, version 4 (GPWv4): Basic demographic characteristics. NASA Socioeconomic Data and Applications Center (SEDAC), accessed 30 November 2019, https://doi.org/10.7927/H49C6VHW. 
Groenemeijer, P., and T. Kühne, 2014: A climatology of tornadoes in Europe: Results from the European Severe Weather Database. Mon. Wea. Rev., 142, 4775-4790, https://doi.org/ 10.1175/MWR-D-14-00107.1.

, and Coauthors, 2017: Severe convective storms in Europe: Ten years of research and education at the European Severe Storms Laboratory. Bull. Amer. Meteor. Soc., 98, 2641-2651, https://doi.org/10.1175/BAMS-D-16-0067.1.

Grünwald, S., and H. E. Brooks, 2011: Relationship between sounding derived parameters and the strength of tornadoes in Europe and the USA from reanalysis data. Atmos. Res., 100, 479-488, https://doi.org/10.1016/j.atmosres.2010.11.011.

Hansen, M. C., and Coauthors, 2013: High-resolution global maps of 21st-century forest cover change. Science, 342, 850-853, https://doi.org/10.1126/science.1244693.

ITU, 2018: International Telecommunications Union Statistics. Accessed 14 November 2018, https://www.itu.int/en/ITU-D/ Statistics/Pages/stat/default.aspx.

Kahraman, A., and P. M. Markowski, 2014: Tornado climatology of Turkey. Mon. Wea. Rev., 142, 2345-2352, https://doi.org/ 10.1175/MWR-D-13-00364.1.

Karpman, D., M. A. R. Ferreira, and C. K. Wikle, 2013: A point process model for tornado report climatology. Stat, 2, 1-8, https://doi.org/10.1002/sta4.14.

Kolobkov, N. V., 1957: Tornado near Moscow (in Russian). Sov. Meteor. Gidrol., 6, 32-33.

Kurgansky, M. V., 2000: The statistical distribution of intense moist-convective, spiral vortices in the atmosphere. Dokl. Earth Sci., 371, 408-410.

— A. V. Chernokulsky, and I. I. Mokhov, 2013: The tornado over Khanty-Mansiysk: An exception or a symptom? Russ. Meteor. Hydrol., 38, 539-546, https://doi.org/10.3103/S1068373913080049.

Letzmann, J., 1923: Die Peipus-Trombe am 3. August 1922. Sitzungsber. d. Naturf. Ges. Univ. Dorpat, 28, 8-42.

Lyakhov, M. Y., 1987: Tornadoes in the midland belt of Russia. Sov. Geogr., 28, 562-570, https://doi.org/10.1080/ 00385417.1987 .10640700 .

Maddison, A., 2010: Statistics on World Population, GDP and Per Capita GDP, 1-2008 AD. Accessed 14 November 2018, http:// www.ggdc.net/MADDISON/oriindex.htm.

Marcinoniene, I., 2003: Tornadoes in Lithuania in the period of 1950-2002 including analysis of the strongest tornado of 29 May 1981. Atmos. Res., 67-68, 475-484, https://doi.org/ 10.1016/S0169-8095(03)00060-7.

McCarthy, D., 2003: NWS tornado surveys and the impact on the national tornado database. First Symp. on the F-Scale and Severe-Weather Damage Assessment, Long Beach, CA, Amer. Meteor. Soc., 3.2, https://ams.confex.com/ams/annual2003/ techprogram/paper_55718.htm.

Meteodnevnik, 2014: Meteorological blog on severe weather. Accessed 14 November 2018, https://vk.com/meteodnevnik2014.

Miglietta, M. M., and I. T. Matsangouras, 2018: An updated "climatology" of tornadoes and waterspouts in Italy. Int. J. Climatol., 38, 3667-3683, https://doi.org/10.1002/joc.5526.

Mikhelson, V., 1904: Hurricane wind in Moscow on 16(29) June 1904 (in Russian). Mon. Meteor. Bull. Main Geophys. Obs., 12, 5-14.

Nalivkin, D. V., 1970: Hurricanes, Storms, Tornadoes (in Russian). Nauka, 488 pp.

NOAA/NWS, 2018: Natural hazard statistics. NOAA/NWS, accessed 14 November 2018, http://www.nws.noaa.gov/om/ hazstats.shtml.

NOAA/NWS Storm Prediction Center, 2018: Warning Coordination Meteorologist's page, daily counts and annual running trend.
NOAA/NWS Storm Prediction Center, accessed 14 November 2018, https://www.spc.noaa.gov/wcm/.

Niino, H., T. Fujitani, and N. Watanabe, 1997: A statistical study of tornadoes and waterspouts in Japan from 1961 to 1993. J. Climate, 10, 1730-1752, https://doi.org/10.1175/1520-0442(1997)010<1730: ASSOTA $>2.0 . \mathrm{CO} ; 2$.

Niva, 1874: Narody Rossii, XXI. Zyryane. Niva, 35, 555-558.

Novitskii, M. A., B. Y. Shmerlin, S. A. Petrichenko, L. A. Tereb, L. K. Kulizhnikova, and O. V. Kalmykova, 2015: Using the indices of convective instability and meteorological parameters for analyzing the tornado-risk conditions in Obninsk on May 23, 2013. Russ. Meteor. Hydrol., 40, 79-84, https://doi.org/ 10.3103/S1068373915020028.

— Y. B. Pavlyukov, B. Y. Shmerlin, S. V. Makhnorylova, N. I. Serebryannik, S. A. Petrichenko, L. A. Tereb, and O. V. Kalmykova, 2016: The tornado in Bashkortostan: The potential of analyzing and forecasting tornado-risk conditions. Russ. Meteor. Hydrol., 41, 683-690, https://doi.org/10.3103/ S1068373916100034.

Okada, R., A. Katsumura, Y. Tamura, M. Matsui, and A. Yoshida, Eds., 2015: Development of Japanese enhanced Fujita scale. Summaries to Technical Papers of Annual Meeting, Japan Association for Wind Engineering, 125-126.

Ovchinnikova, R., 2013: Tornadoes in Belarus. Accessed 14 November 2018, http://www.geoversum.by/blogs/tapanm-ka/smerchi-v-belarusi.html.

Peryshkin, I. N., 1957: A tornado in Belorussia (in Russian). Sov. Meteor. Gidrol., 5, 29-30.

Piyp, B. I., 2005: A secret tsunami (in Russian). Priroda, 1077, 37-44.

Popov, B. M., 1954: Passage of a tornado through Rostov, Yaroslavl region on August 24, 1953 (in Russian). Sov. Meteor. Gidrol., 3, 27-30.

Popov, N. I., 1955: Tornadoes at the Black Sea coast (in Russian). Sov. Meteor. Gidrol., 5, 35-37.

Prokh, L. Z., 1983: Tales on Winds (in Russian). Radyanska Shkola, $192 \mathrm{pp}$.

and Z. M. Skotarenko, 1971: Tornadoes in the Dnepr region (in Russian). Sov. Meteor. Gidrol., 2, 105-109.

Rauhala, J., H. E. Brooks, and D. M. Schultz, 2012: Tornado climatology of Finland. Mon. Wea. Rev., 140, 1446-1456, https:// doi.org/10.1175/MWR-D-11-00196.1.

Romanskii, S. O., E. M. Verbitskaya, S. V. Ageeva, and D. P. Istomin, 2018: Tornado in the city of Blagoveshchensk on July 31, 2011. Russ. Meteor. Hydrol., 43, 574-580, https://doi.org/ 10.3103/S1068373918090030.

Schaefer, J. T., D. L. Kelly, and R. F. Abbey, 1986: A minimum assumption tornado-hazard probability model. J. Climate Appl. Meteor., 25, 1934-1945, https://doi.org/10.1175/15200450(1986)025<1934:AMATHP>2.0.CO;2.

Schultz, D. M., Y. P. Richardson, P. M. Markowski, and C. A. Doswell III, 2014: Tornadoes in the central United States and the "Clash of air masses." Bull. Amer. Meteor. Soc., 95, 17041712, https://doi.org/10.1175/BAMS-D-13-00252.1.

Scientific and Engineering Centre for Nuclear and Radiation Safety, 2001: Recommendations to assessment of tornado characteristics in respect to nuclear facilities (in Russian). Rep. RB-022-01. Ordinance of Gosatomnadzor of Russia, No. 17, 59-90.

Shikhov, A., 2013: The study of impacts of severe convective storms in Perm region, using earth remote sensing data (in Russian). Geograficheskiy Vestn., 24, 77-86.

, and A. Chernokulsky, 2018: A satellite-derived climatology of unreported tornadoes in forested regions of northeast Europe. Remote Sens. Environ., 204, 553-567, https://doi.org/ 10.1016/j.rse.2017.10.002. 
,-- A. Sprygin, and I. Azhigov, 2019: Identification of mesoscale convective cloud systems with tornadoes using satellite data (in Russian). Sovrem. Probl. Distantsionnogo Zondirovaniya Zemli Kosmosa, 16, 223-236, https://doi.org/ 10.21046/2070-7401-2019-16-1-223-236.

Silverman, B. W., 1986: Density Estimation for Statistics and Data Analysis. 1st ed. Chapman and Hall, 175 pp.

Simeonov, P., L. Bocheva, and I. Gospodinov, 2013: On space-time distribution of tornado events in Bulgaria (1956-2010) with brief analyses of two cases. Atmos. Res., 123, 61-70, https:// doi.org/10.1016/j.atmosres.2012.07.003.

Sioutas, M. V., and A. G. Keul, 2007: Waterspouts of the Adriatic, Ionian and Aegean Sea and their meteorological environment. Atmos. Res., 83, 542-557, https://doi.org/10.1016/j.atmosres.2005.08.009.

Snitkovskii, A., 1987: Tornadoes in the USSR (in Russian). Sov. Meteor. Gidrol., 9, 12-25.

Starov, D. K., 1935: Waterspouts over the Black and Azov Seas (in Russian). Notes Hydrogr., 1, 56-58.

Taszarek, M., and H. E. Brooks, 2015: Tornado climatology of Poland. Mon. Wea. Rev., 143, 702-717, https://doi.org/10.1175/ MWR-D-14-00185.1.

- and J. Gromadzki, 2017: Deadly tornadoes in Poland from 1820 to 2015. Mon. Wea. Rev., 145, 1221-1243, https://doi.org/ 10.1175/MWR-D-16-0146.1.

—_, and Coauthors, 2019: A climatology of thunderstorms across Europe from a synthesis of multiple data sources. J. Climate, 32, 1813-1837, https://doi.org/10.1175/JCLI-D-18-0372.1.

Tippett, M. K., J. T. Allen, V. A. Gensini, and H. E. Brooks, 2015: Climate and hazardous convective weather. Curr. Climate Change Rep., 1, 60-73, https://doi.org/10.1007/s40641015-0006-6.

- C. Lepore, and J. E. Cohen, 2016: More tornadoes in the most extreme US tornado outbreaks. Science, 354, 1419-1423, https://doi.org/10.1126/science.aah7393.

Tomashevich, L. V., 1952: A tornado in Tula on 4 June 1948 (in Russian). Sov. Meteor. Gidrol., 4, 23-26.

Tooming, H., and M. Merilain, 2004: A tornado map for Estonia. J. Meteor., 29 (286), 51-57.

Tornado list, 2013: Catalogue of tornadoes in the Commonwealth of Independent States in 2013. Accessed 14 November 2018, https://yandex.ru/maps/-/CCeLUK4d.

, 2014: Catalogue of tornadoes in the Commonwealth of Independent States in 2014. Accessed 14 November 2018, https://yandex.ru/maps/-/CCeLUHLv.
- 2015: Catalogue of tornadoes in the Commonwealth of Independent States in 2015. Accessed 14 November 2018, https://yandex.ru/maps/-/CCeLUUnD.

- 2016: Catalogue of tornadoes in the Commonwealth of Independent States in 2016. Accessed 14 November 2018, https://yandex.ru/maps/-/CCeLYYKY.

TTU, 2004: A Recommendation for an Enhanced Fujita scale (EF-Scale). Texas Tech University, 95 pp.

Tunegolovetc, V. P., and T. D. Mikhailenko, 1998: Tornadoes in Vladivostok on 20 September 1997 (in Russian). Russ. Meteor. Hydrol., 6, 100-107.

Vashchenko, V. M., I. B. Korduba, Y. A. Loza, Z. I. Patlashenko, O. O. Bannikov, and Y. M. Kryzska, 2018: Tornado statistics in Ukraine based on new data. Geofizicheskiy Z., 40, 199-213, https://doi.org/10.24028/gzh.0203-3100.v40i3.2018.137206.

Vasiliev, A. A., B. E. Peskov, and A. Snitkovskii, 1985: Whirlwinds, squalls and hail of 8-9 June 1984 (in Russian). Sov. Meteor. Gidrol., 8, 5-15.

Verbout, S. M., H. E. Brooks, L. M. Leslie, and D. M. Schultz, 2006: Evolution of the U.S. tornado database: 1954-2003. Wea Forecasting, 21, 86-93, https://doi.org/10.1175/WAF910.1.

Virts, K. S., J. M. Wallace, M. L. Hutchins, and R. H. Holzworth, 2013: Highlights of a new ground-based, hourly global lightning climatology. Bull. Amer. Meteor. Soc., 94, 1381-1391, https://doi.org/10.1175/BAMS-D-12-00082.1.

Vivchar, A., 2011: Wildfires in Russia in 2000-2008: Estimates of burnt areas using the satellite MODIS MCD45 data. Remote Sens. Lett., 2, 81-90, https://doi.org/10.1080/01431161.2010.499138.

Voznyachuk, L. N., 1954a: Materials for the study of tornadoes in the territory of Belarus (in Russian). Bull. Belarus. State Univ., 21, 104-123.

- 1954b: On the question of determining the northern boundary of the spread of tornadoes in Europe and Western Siberia (in Russian). Bull. Belarus. State Univ., 21, 87-103.

Wurman, J., J. M. Straka, and E. N. Rasmussen, 1996: Fine-scale Doppler radar observations of tornadoes. Science, 272, 17741777, https://doi.org/10.1126/science.272.5269.1774.

—- D. Dowell, Y. Richardson, P. Markowski, E. Rasmussen, D. Burgess, L. Wicker, and H. B. Bluestein, 2012: The second Verification of the Origins of Rotation in Tornadoes Experiment: VORTEX2. Bull. Amer. Meteor. Soc., 93, 1147-1170, https://doi.org/10.1175/BAMS-D-11-00010.1.

Zykov, I. V., 1947: Tornadoes in the Western Siberia (in Russian). Priroda, 11, 54-55. 